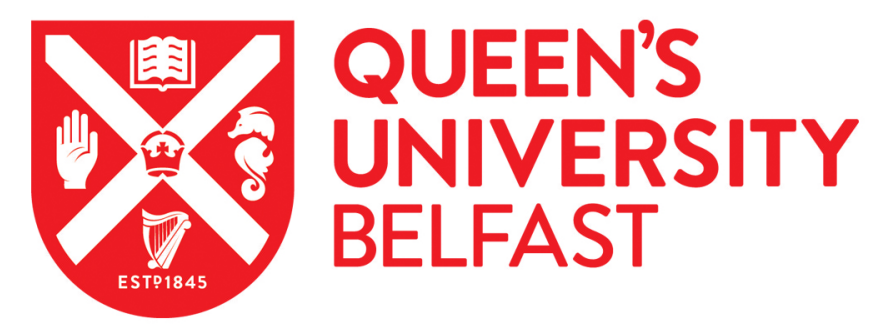

\title{
NOMA Throughput and Energy Efficiency in Energy Harvesting Enabled Networks
}

Nasir, A. A., Tuan, H. D., Duong, T. Q., \& Debbah, M. (2019). NOMA Throughput and Energy Efficiency in Energy Harvesting Enabled Networks. IEEE Transactions on Communications.

https://doi.org/10.1109/TCOMM.2019.2919558

\section{Published in:}

IEEE Transactions on Communications

\section{Document Version:}

Peer reviewed version

\section{Queen's University Belfast - Research Portal:}

Link to publication record in Queen's University Belfast Research Portal

\section{Publisher rights}

(C) 2019, IEEE.

This work is made available online in accordance with the publisher's policies. Please refer to any applicable terms of use of the publisher.

\section{General rights}

Copyright for the publications made accessible via the Queen's University Belfast Research Portal is retained by the author(s) and / or other copyright owners and it is a condition of accessing these publications that users recognise and abide by the legal requirements associated with these rights.

Take down policy

The Research Portal is Queen's institutional repository that provides access to Queen's research output. Every effort has been made to ensure that content in the Research Portal does not infringe any person's rights, or applicable UK laws. If you discover content in the Research Portal that you believe breaches copyright or violates any law, please contact openaccess@qub.ac.uk. 


\title{
NOMA Throughput and Energy Efficiency in Energy Harvesting Enabled Networks
}

\author{
Ali A. Nasir, Hoang D. Tuan, Trung Q. Duong, and Merouane Debbah
}

\begin{abstract}
An energy harvesting (EH) enabled network is capable of delivering energy to users, who are located sufficiently close to the base stations. However, the wireless energy delivery requires much more transmit power than what the normal information delivery does. It is very challenging to provide the quality of the wireless information and power delivery simultaneously. It is of practical interest to employ nonorthogonal multiple access (NOMA) to improve the network throughput, while fulfilling the $\mathrm{EH}$ requirements. To realize both EH and information decoding, this paper considers a transmit time-switching (transmit-TS) protocol. Two important problems of users' max-min throughput optimization and energy efficiency maximization under power constraint and EH thresholds, which are non-convex in beamforming vectors, are addressed by efficient path-following algorithms. In addition, the conventional power splitting (PS)-based EH receiver is also considered. The provided numerical results confirm that the proposed transmit-TS based algorithms clearly outperform the PS-based algorithms in terms of both, throughput and energy efficiency.
\end{abstract}

Index Terms-Wireless power delivery, energy harvesting, non-orthogonal multiple access (NOMA), transmit timeswitching, nonconvex optimization, throughput, energy efficiency, quality-of-service $(\mathrm{QoS})$.

\section{INTRODUCTION}

\section{A. Motivation}

Radio frequency (RF) energy harvesting (EH) [1]-[3] has emerged as a potential technology to energize the Internet of things. To realize both wireless $\mathrm{EH}$ and information decoding (ID), the users need to split the received signal for EH and ID either by power splitting (PS) or time switching (TS), where the latter is referred to as "receive-TS" [1], [4], [5]. PS has been shown mostly outperforming receive-TS

This work was supported in part by Vietnam National Foundation for Science and Technology Development (NAFOSTED) under Grant No. 102.04-2017.301, in part by the KFUPM Research Project \#SB171005, in part by the Australian Research Council's Discovery Projects under Project DP190102501, and in part by the U.K. Royal Academy of Engineering Research Fellowship under Grant RF1415 $\backslash 14 \backslash 22$.

Ali A. Nasir is with the Department of Electrical Engineering, King Fahd University of Petroleum and Minerals (KFUPM), Dhahran, Saudi Arabia (email: anasir@kfupm.edu.sa)

Hoang D. Tuan is with the School of Electrical and Data Engineering, University of Technology, Sydney, NSW 2007, Australia (email: tuan.hoang@uts.edu.au).

Trung Q. Duong is with the School of Electronics, Electrical Engineering and Computer Science, Queen's University Belfast, Belfast BT7 1NN, United Kingdom (e-mail: trung.q.duong@qub.ac.uk).

Merouane Debbah is with the Mathematical and Algorithmic Sciences Laboratory, France Research Center, Huawei Technologies, France (email: merouane.debbah@huawei.com). but it is complicated and inefficient for practical implementation. Recent findings in [4], [6] and [7] demonstrate the advantages of the new "transmit-TS" approach over the PS approach, where information and energy are transferred separately so the users do not need any sophisticated device for $\mathrm{EH}$.

For information transmission, one of the most critical tasks is to provide the quality of service (QoS) in terms of throughput to those users, who are located far from the BS. Non-orthogonal multiple access (NOMA) technique (see e.g. [8] and [9]) is able to improve the throughput at these users by allowing the near-by users to access the information intended for them. NOMA is also capable of supporting numbers of users that are more than the number of available orthogonal time-, frequency-, or code-domain resources, so it is considered as a potential candidate to satisfy the radical spectral efficiency and massive connectivity requirements of 5G [10]. An efficient beamforming design for NOMA multicell systems was proposed in [11].

\section{B. Literature Survey}

A wireless powered communication network, where the BS charges users and enables them to transmit information for uplink communication by using NOMA, was considered in [12] and [13]. A similar study for a wireless-powered sensor network was conducted in [14]. The trade-off among the energy efficiency, fairness, harvested energy, and system sum rate of a NOMA based heterogeneous network was investigated in [15]. Optimal resource allocation strategies for cognitive radio networks with NOMA were designed in [16]. Wireless power transfer and NOMA in machine-to-machine communication, where the machine-type communication device harvests energy in the downlink while transmitting information to the BS via machine-type communication gateway in the uplink, were considered [17] and [18]. All these works considered problems for single antenna nodes, which either harvest energy or decode information but not implement both $\mathrm{EH}$ and ID.

In EH based cooperative NOMA systems, the cellcentered or "nearly-located users" harvest energy from the wireless signals received from the BSs and act as a relay to forward the information to the "far-located users". Most of works used PS at the relay users. Outage expressions for far end users were derived in [19]-[22]. Different users 
pairing schemes for NOMA were proposed in [23]. The problem of maximizing the data rate of the strong user while satisfying the QoS requirement of the weak user was considered in [24]. Antenna selection schemes at the BS were proposed in [21].

A hybrid receive-TS/PS for EH at the relay users was proposed in a recent work [25], while a simple TS mode at the relay receiver, where half of the time is dedicated for $\mathrm{EH}$, was considered in [26].

Exploiting wireless EH in NOMA systems, the work [27] considered various energy harvesting protocols, e.g., conventional receive-TS, PS and generalized (hybrid TS/PS) under a setup of a single antenna BS and a single user pair. Wireless power transfer by PS and information transmission in a NOMA system was also studied in [28] under a simple setup with a single antenna BS. The work [29] considered an energy-constrained full-duplex information transmitter, which harvests energy from a dedicated energy transmitter while transmitting information to the information receivers by employing NOMA. A cognitive radio based wireless information and power transfer network with NOMA was considered in [30].

\section{Research Gap and Contribution}

Recently, throughput optimization with multi-antenna beamforming and PS based EH in simple NOMA systems with one BS serving one nearly-located user and one farlocated user, was addressed in [24]. Like [29] and [30], semidefinite relaxation (SDR) is its tool for computation, which is quite inefficient because SDR has to first solve for matrix optimization of much higher dimension [6], [31]. In addition, it employed a PS-based approach for EH, which is also not very practical due to the need of variable PS. Thus, an efficient solution for such important throughput max-min optimization problem is still missing. Meanwhile, the energy efficiency (EE), which is defined as the sum throughput per Joule of consumed energy (ratio of sum throughput to power consumption), is a key performance indicator for new wireless technologies [32]. Therefore, EE maximization is an important target for EH enabled NOMA systems. To the best of authors' knowledge, the problem of EE maximization by multi-antenna beamforming in $\mathrm{EH}$ enabled NOMA network has not been addressed in the literature. This problem is more computationally difficult than the throughput maximization problem due to the additional optimization variables appearing in the denominator of the EE function [33], [34].

This paper considers multi-antenna beamforming in an EH enabled NOMA network, which delivers information and energy separately in fractional times, enabling the EH by simple devices. Thus, both throughput and harvested energy can be improved by separated information and energy beamformers. We formulate two important problems of throughput max-min optimization and EE maximization under power constraint and EH constraints at the nearly- located users. As these problems are highly nonconvex, checking their feasibility is already computationally difficult. Nevertheless, like [6], [7] for throughput max-min optimization and EE in orthogonal multiple access (OMA) EH enabled networks, and [11] for throughput maximization in NOMA networks, we aim to develop efficient pathfollowing algorithms for their computation. Compared to these our previous works, the contribution of the paper is two-fold:

- To address the problem of throughput max-min optimization with new and much more complex nonconvex constraints compared with those in [6], [7], [11], we propose completely new their inner convex approximations. They lead to path-following algorithms of low-computational complexity, which converge rapidly to an optimal solution.

- To address the problem of EE maximization, we also develop new path-following computational procedures, which avoid approximations of the entire EE objective functions proposed in [7]. As such, they reveal that this problem is not more computationally complex than the problem of throughput maximization, contrary to the commonly accepted conception [33], [34]

For comparison purpose, we consider both PS-based and transmit-TS based energy harvesting and propose novel algorithms to solve above-mentioned novel beamforming problems. Our numerical results confirm that the proposed transmit-TS approach clearly outperforms the PS approach in terms of both, throughput and energy efficiency.

\section{Organization and Notation}

The paper is organized as follows. After a brief introduction of system model, Section II presents the formulation of throughput and EE maximization problems and their computational solution for PS-based NOMA implementation. Section III describes the formulation and solution of such problems for transmit-TS based NOMA implementation. Section IV evaluates the performance of our proposed algorithms by numerical examples. Finally, Section V concludes the paper.

Notation. Bold-faced lower-case letters, e.g., $\mathrm{x}$, are used for vectors and lower-case letters, e.g., $x$, are used for for scalars. $\mathbf{x}^{H}, \mathbf{x}^{T}$, and $\mathbf{x}^{*}$ denote Hermitian transpose, normal transpose, and conjugate of the vector $\mathbf{x}$, respectively. $\|\cdot\|$ stands for the vector's Euclidean norm. $\mathbb{C}$ is the set of all complex numbers, and $\emptyset$ is an empty set. $\Re\{x\}$ denotes the real part of a complex number $x . \nabla f(\mathbf{x})$ is the gradient of function $f(\cdot)$ at $\mathbf{x}$. Also, we define $\langle\mathbf{x}, \mathbf{y}\rangle \triangleq \mathbf{x}^{H} \mathbf{y}$. $\operatorname{Col}\left[a_{i}\right]_{i \in \mathcal{I}}$ arranges $a_{i}, i \in \mathcal{I}$ in row-block. For instance

$$
\operatorname{Col}\left[a_{i}\right]_{i \in\{1,2\}}=\left[\begin{array}{l}
a_{1} \\
a_{2}
\end{array}\right] .
$$




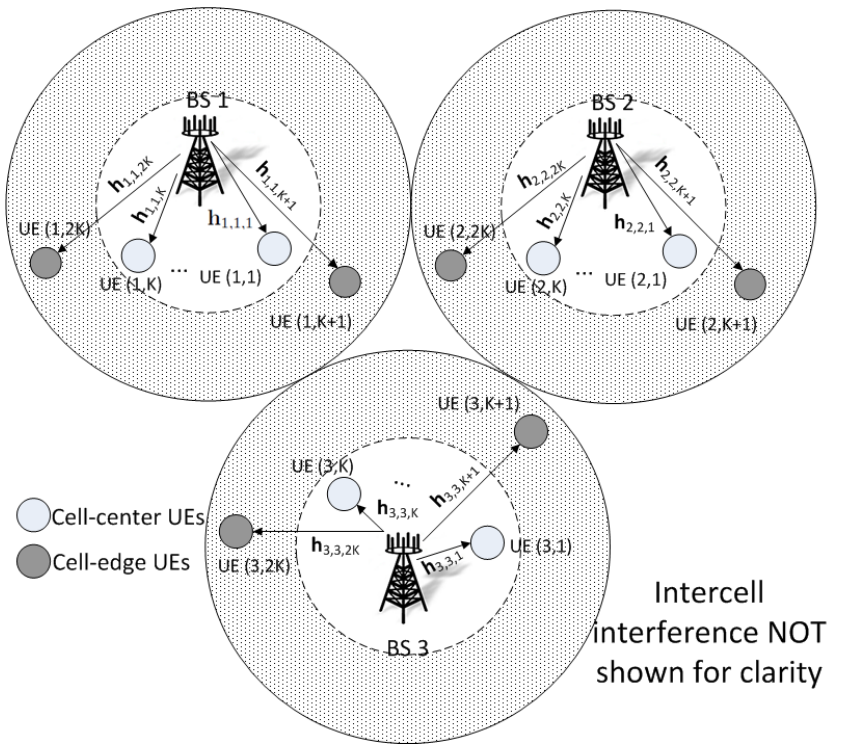

Fig. 1. Downlink multiuser multicell interference scenario in a dense network consisting of $K$ small cells. For clarity, the intercell interference channels are not shown, however, the interference occurs in all $K$ cells.

\section{SySTEM MODEL AND PS-BASED NOMA}

Consider a downlink system consisting of $N$ cells under dense deployment, where the BS of each cell is equipped with $N_{t}$ antennas to serve $2 K$ single-antenna-equipped users (UEs) within its cell. In each cell, there are $K$ near UEs (cell-center UEs), which are located inside the inner circular area and $K$ far UEs (cell-edge UEs), which are located in the ring area between inner circle and outer radius. A representative figure for three cells is shown in Fig. 1. The $K$ far UEs in each cell are not only in poorer channel conditions than other $K$ near UEs but also are under more drastic inter-cell interference from adjacent cells.

Upon denoting $\mathcal{I} \triangleq\{1,2, \cdots, N\}$ and $\mathcal{J} \triangleq$ $\{1,2, \cdots, 2 K\}$, the $j$-th UE in the $i$-th cell is referred to as UE $(i, j) \in \mathcal{S} \triangleq \mathcal{I} \times \mathcal{J}$. The cell-center UEs are UE $(i, j), j \in \mathcal{J}_{c} \triangleq\{1, \cdots, K\}$ while the cell-edge UEs are $\mathrm{UE}(i, j), j \in \mathcal{J}_{e} \triangleq\{K+1, \cdots, 2 K\}$. Thus the set of cellcenter UEs and the set of cell-edge UEs are $\mathcal{S}_{c} \triangleq \mathcal{I} \times \mathcal{J}_{c}$ and $\mathcal{S}_{e} \triangleq \mathcal{I} \times \mathcal{J}_{e}$, respectively. Due their proximity, the cell-center $\mathrm{UE}(i, j), j \in \mathcal{J}_{c}$ is able to do both information decoding and energy harvesting. By exploiting the differentiated channel conditions between the cell-center and celledge UEs, each cell-center UE $(i, j) \in \mathcal{S}_{c}$ is randomly paired with cell-edge $\mathrm{UE}(i, p(j)) \in \mathcal{S}_{e}$ of the same cell to create a virtual cluster to improve the network throughput. ${ }^{1}$ For notational convenience, the paired UE $(i, p(j))$ for UE $(i, j)$ is chosen, such that $p(j)=j+K$.

\footnotetext{
${ }^{1}$ This is a practical user-pairing strategy. Using more sophisticated user-pairing strategies may improve the performance of MIMO-NOMA networks (see e.g. [35]) but this is beyond the scope of this paper.
}

\section{A. PS-based NOMA}

For comparison point-of-view with transmit-TS based NOMA, which will be presented in Section III, let us first develop the system model, problem formulation, and solution approach for PS-based NOMA implementation.

The signal superpositions are precoded at the BSs prior to being transmitted to the UEs. Specifically, the message intended for UE $(i, j)$ is $s_{i, j} \in \mathbb{C}$ with $\mathbb{E}\left\{\left|s_{i, j}\right|^{2}\right\}=1$, which is beamformed by vector $\mathbf{w}_{i, j} \in \mathbb{C}^{N_{t}}$. The received signals at UE $(i, j)$ and $\mathrm{UE}(i, p(j))$ are expressed as

$$
y_{i, j}=\sum_{(s, \ell) \in \mathcal{S}} \mathbf{h}_{s, i, j} \mathbf{w}_{s, \ell} s_{s, \ell}+n_{i, j}
$$

and

$$
y_{i, p(j)}=\sum_{(s, \ell) \in \mathcal{S}} \mathbf{h}_{s, i, p(j)} \mathbf{w}_{s, \ell} s_{s, \ell}+n_{i, p(j)},
$$

where $\mathbf{h}_{s, i, j} \in \mathbb{C}^{1 \times N_{t}}$ is the MISO channel from the BS $s \in \mathcal{I}$ to $\operatorname{UE}(i, j) \in \mathcal{S}$ and $n_{i, j} \sim \mathcal{C N}(0, \sigma)$ is the additive noise. In this paper, we assume that full channel state information is available by some means, e.g., through coordination among the BSs [36].

To implement simultaneous wireless information and power transfer (SWIPT), the power splitter divides the received signal $y_{i, j}$ into two parts in the proportion of $\alpha_{i, j}:\left(1-\alpha_{i, j}\right)$, where $\alpha_{i, j} \in(0,1)$ is termed as the PS ratio for UE $(i, j)$. The first part $\sqrt{\alpha_{i, j}} y_{i, j}$ forms an input to the ID receiver as:

$$
\begin{array}{r}
\sqrt{\alpha_{i, j}} y_{i, j}+z_{i, j}^{c}= \\
\sqrt{\alpha_{i, j}}\left(\sum_{(s, \ell) \in \mathcal{S}} \mathbf{h}_{s, i, j} \mathbf{w}_{s, \ell} s_{s, \ell}+n_{i, j}\right)+z_{i, j}^{c},
\end{array}
$$

where $z_{i, j}^{c} \sim \mathcal{C N}\left(0, \sigma_{c}\right)$ is the additional noise introduced by the ID receiver circuitry.

The energy of the second part $\sqrt{1-\alpha_{i, j}} y_{i, j}$ of the received signal $y_{i, j}$ is harvested by the EH receiver of UE $(i, j)$ as

$$
E_{i, j}\left(\mathbf{w}, \alpha_{i, j}\right) \triangleq \zeta_{i, j}\left(1-\alpha_{i, j}\right)\left(p_{i, j}(\mathbf{w})\right) .
$$

For notational convenience, we define $\mathbf{w} \triangleq\left[\mathbf{w}_{i, j}\right]_{(i, j) \in \mathcal{S}}$, which constitutes all possible beamforming vectors. As can be sensed from (3) and (4), the near-by UEs $(i, j)$ harvest energy through wireless signals not only from the serving BSs but also from the neighboring BSs. Note that the harvested and stored energy $E_{i, j}$ may be used later for different power constrained operations at cell-center UEs $(i, j)$, e.g., assisting uplink data transmission to the BS or performing downlink information processing. In (4), the constant $\zeta_{i, j} \in(0,1)$ denotes the efficiency of energy conversion at the $\mathrm{EH}$ receiver, and

$$
p_{i, j}(\mathbf{w})=\sum_{(s, \ell) \in \mathcal{S}}\left|\mathbf{h}_{s, i, j} \mathbf{w}_{s, \ell}\right|^{2} .
$$


In NOMA, the message $s_{i, p(j)}$ is decoded by UE $(i, j)$ and $(i, p(j))$. The interference plus noise at $\operatorname{UE}(i, j)$ in decoding $s_{i, p(j)}$ is

$$
\begin{aligned}
\mathbb{I N}_{i, p(j)}^{c}(\mathbf{w}) \triangleq & \alpha_{i, j}\left(\sum_{(s, \ell) \in \mathcal{S} \backslash\{(i, p(j))\}}\left|\mathbf{h}_{s, i, j} \mathbf{w}_{s, \ell}\right|^{2}\right. \\
& +\sigma)+\sigma_{c} \\
= & \alpha_{i, j}\left(\left\|\mathcal{L}_{i, p(j)}^{c}(\mathbf{w})\right\|^{2}+\sigma\right)+\sigma_{c},
\end{aligned}
$$

with

$$
\mathcal{L}_{i, p(j)}^{c}(\mathbf{w}) \triangleq \operatorname{Col}\left[\mathbf{h}_{s, i, j} \mathbf{w}_{s, \ell}\right]_{(s, \ell) \in \mathcal{S} \backslash\{(i, p(j))\}},
$$

which is a linear operator. Therefore, the signal-tointerference-plus-noise (SINR) in decoding $s_{i, p(j)}$ at UE $(i, j)$ is $\left|\mathbf{h}_{i, i, j} \mathbf{w}_{i, p(j)}\right|^{2} /\left(\left\|\mathcal{L}_{i, p(j)}^{c}(\mathbf{w})\right\|^{2}+\sigma+\sigma_{c} / \alpha_{i, j}\right)$.

Meanwhile, the edge-user UE $(i, p(j)$ decodes its own message $s_{i, p(j)}$ only, so the interference plus noise at UE $(i, p(j))$ is $I \mathrm{~N}_{i, p(j)}^{e}(\mathbf{w}) \triangleq\left\|\mathcal{L}_{i, p(j)}^{e}(\mathbf{w})\right\|^{2}+\sigma$ with the linear operator

$$
\mathcal{L}_{i, p(j)}^{e}(\mathbf{w}) \triangleq \operatorname{Col}\left[\mathbf{h}_{s, i, p(j)} \mathbf{w}_{s, \ell}\right]_{(s, \ell) \in \mathcal{S} \backslash\{(i, p(j))\}} .
$$

In what follows, we use the general rate function $\psi(\mathbf{x}, \mathbf{y}, \nu)$ defined by

$$
\psi(\mathbf{x}, \mathbf{y}, \nu)=\ln \left(1+\frac{\|\mathbf{x}\|^{2}}{\|\mathbf{y}\|^{2}+\sigma+\sigma_{c} \nu}\right) .
$$

Suppose that $\mathcal{R}_{i, p(j)}$ is the achievable rate in decoding $s_{i, p(j)}$. Since both near UE $(i, j)$ and far UE $(i, p(j))$ will decode the far user's information $s_{i, p(j)}$, thus, $\mathcal{R}_{i, p(j)}$ for $\mathrm{UE}(i, j)$ and $\mathrm{UE}(i, p(j))$, respectively, is defined by

$$
\begin{array}{r}
\min \left\{\psi\left(\mathbf{h}_{i, i, j} \mathbf{w}_{i, p(j)}, \mathcal{L}_{i, p(j)}^{c}(\mathbf{w}), 1 / \alpha_{i, j}\right),\right. \\
\left.\psi\left(\mathbf{h}_{i, i, j} \mathbf{w}_{i, p(j)}, \mathcal{L}_{i, p(j)}^{e}(\mathbf{w}), 0\right)\right\} .
\end{array}
$$

Thus $\mathcal{R}_{i, p(j)}$ satisfies the following constraints:

$$
\begin{aligned}
& \mathcal{R}_{i, p(j)} \leq \psi\left(\mathbf{h}_{i, i, j} \mathbf{w}_{i, p(j)}, \mathcal{L}_{i, p(j)}^{c}(\mathbf{w}), 1 / \alpha_{i, j}\right), \\
& \mathcal{R}_{i, p(j)} \leq \psi\left(\mathbf{h}_{i, i, p(j)} \mathbf{w}_{i, p(j)}, \mathcal{L}_{i, p(j)}^{e}(\mathbf{w}), 0\right) .
\end{aligned}
$$

UE $(i, j)$ subtracts $s_{i, p(j)}$ from the right hand side of (3) in decoding $s_{i, j}$. Then the achievable rate $\mathcal{R}_{i, j}$ by decoding $s_{i, j}$ is $\psi\left(\mathbf{h}_{i, i, j} \mathbf{w}_{i, j}, \mathcal{L}_{i, j}^{c}(\mathbf{w}), 1 / \alpha_{i, j}\right)$ and is expressed by the constraint:

$$
\mathcal{R}_{i, j} \leq \psi\left(\mathbf{h}_{i, i, j} \mathbf{w}_{i, j}, \mathcal{L}_{i, j}^{c}(\mathbf{w}), 1 / \alpha_{i, j}\right),
$$

with

$$
\mathcal{L}_{i, j}^{c}(\mathbf{w}) \triangleq \operatorname{Col}\left[\mathbf{h}_{s, i, j} \mathbf{w}_{s, \ell}\right]_{(s, \ell) \in \mathcal{S} \backslash\{(i, p(j)),(i, j)\}} .
$$

For convenience, we also use the notations

$$
\begin{gathered}
\alpha \triangleq\left(\alpha_{i, j}\right)_{(i, j) \in \mathcal{S}} \\
\mathcal{R} \triangleq\left(\mathcal{R}_{i, j}\right)_{(i, j) \in \mathcal{S}} .
\end{gathered}
$$

We consider two basic problems:

\section{1) Throughput max-min optimization}

$$
\begin{array}{r}
\max _{\mathbf{w}, \mathcal{R}, \boldsymbol{\alpha}} \min _{(i, j) \in \mathcal{I} \times \mathcal{J}} \mathcal{R}_{i, j} \quad \text { s.t. } \quad(9),(10),(11), \\
\zeta_{i, j}\left(p_{i, j}(\mathbf{w})\right) \geq \frac{e_{i, j}^{\min }}{1-\alpha_{i, j}}, i \in \mathcal{I}, j \in \mathcal{J}_{c}, \\
0<\alpha_{i, j}<1, i \in \mathcal{I}, j \in \mathcal{J}_{c}, \\
\sum_{j \in \mathcal{J}}\left\|\mathbf{w}_{i, j}\right\|^{2} \leq P_{i}^{\max }, i \in \mathcal{I},
\end{array}
$$

where (13b) defines the EH constraint such that $e_{i, j}^{\min }$ is the EH threshold and $p_{i, j}(\mathbf{w})$ is defined in (5), and $P_{i}^{\max }$ in (13d) is the transmit power budget of BS $i$.

2) Energy-efficiency maximization under QoS constraints

$$
\begin{aligned}
& \max _{\mathbf{w}, \mathcal{R}, \boldsymbol{\alpha}} \mathcal{F}(\mathbf{w}, \mathcal{R}) \triangleq \frac{\sum_{(i, j) \in \mathcal{I} \times \mathcal{J}} \mathcal{R}_{i, j}}{\xi \pi(\mathbf{w})+P_{c}} \\
& \text { s.t. } \quad(9),(10),(11),(13 b),(13 c),(13 d), \\
& \mathcal{R}_{i, j} \geq r_{i, j}, i \in \mathcal{I}, j \in \mathcal{J}_{c}, \\
& \mathcal{R}_{i, p(j)} \geq r_{i, p(j)}, i \in \mathcal{I}, j \in \mathcal{J}_{c},
\end{aligned}
$$

where $\pi(\mathbf{w})=\sum_{i \in \mathcal{I}} \sum_{j \in \mathcal{J}}\left\|\mathbf{w}_{i, j}\right\|^{2}, \xi$ is the reciprocal constant power amplifier efficiency, $P_{c} \triangleq N_{t} P_{A}+P_{\text {cir }}, P_{A}$ is the power dissipation at each transmit antenna, $P_{\text {cir }}$ is the fixed circuit power consumption for base-band processing, and (14b) and (14c) are the QoS constraints, such that, $r_{i, j}$, $i \in \mathcal{I}, j \in \mathcal{J}$ is the threshold rate to ensure a certain QoS.

\section{B. Computational solutions for PS-based NOMA}

Let us first address the throughput max-min optimization problem (13). We have to resolve the non-convex rate constraints (9), (10), and (11) and the non-convex EH constraint (13b). In order to deal with the non-convexity of rate constraints (9), (10), and (11), we have to provide a concave lower bounding function for $\psi(\mathbf{x}, \mathbf{y}, 0)$ defined by (8), at a given point $\left(\mathbf{x}^{(\kappa)}, \mathbf{y}^{(\kappa)}\right)$ [5], [7]. In the Appendix A, we prove the following new and universal concave function bound:

$$
\psi(\mathbf{x}, \mathbf{y}, \mu) \geq \Lambda(\mathbf{x}, \mathbf{y}, \mu)
$$

over the trust region

$$
2 \Re\left\{\left(\mathbf{x}^{(\kappa)}\right)^{H} \mathbf{x}\right\}-\left\|\mathbf{x}^{(\kappa)}\right\|^{2}>0,
$$

with $\psi\left(\mathbf{x}^{(\kappa)}, \mathbf{y}^{(\kappa)}, \mu^{(\kappa)}\right)=\Lambda\left(\mathbf{x}^{(\kappa)}, \mathbf{y}^{(\kappa)}, \mu^{(\kappa)}\right)$, where

$$
\begin{aligned}
\Lambda(\mathbf{x}, \mathbf{y}, \mu) \triangleq & a\left(\mathbf{x}^{(\kappa)}, \mathbf{y}^{(\kappa)}, \mu^{(\kappa)}\right) \\
& -\frac{\left\|\mathbf{x}^{(\kappa)}\right\|^{2}}{2 \Re\left\{\left(\mathbf{x}^{(\kappa)}\right)^{H} \mathbf{x}\right\}-\left\|\mathbf{x}^{(\kappa)}\right\|^{2}} \\
& -b\left(\mathbf{x}^{(\kappa)}, \mathbf{y}^{(\kappa)}, \mu^{(\kappa)}\right)\|\mathbf{x}\|^{2} \\
& -c\left(\mathbf{x}^{(\kappa)}, \mathbf{y}^{(\kappa)}, \mu^{(\kappa)}\right)\left(\|\mathbf{y}\|^{2}+\sigma_{c} \mu\right),
\end{aligned}
$$

and

$$
\begin{aligned}
& a\left(\mathbf{x}^{(\kappa)}, \mathbf{y}^{(\kappa)}, \mu^{(\kappa)}\right)=\psi\left(\mathbf{x}^{(\kappa)}, \mathbf{y}^{(\kappa)}, \mu^{(\kappa)}\right)+2 \\
& -\frac{\left\|\mathbf{x}^{(\kappa)}\right\|^{2}}{\left\|\mathbf{x}^{(\kappa)}\right\|^{2}+\left\|\mathbf{y}^{(\kappa)}\right\|^{2}+\tilde{\mu}^{(\kappa)}} \frac{\sigma}{\left\|\mathbf{y}^{(\kappa)}\right\|^{2}+\tilde{\mu}^{(\kappa)}},
\end{aligned}
$$




$$
\begin{aligned}
& <b\left(\mathbf{x}^{(\kappa)}, \mathbf{y}^{(\kappa)}, \mu^{(\kappa)}\right) \\
& =\frac{\left\|\mathbf{y}^{(\kappa)}\right\|^{2}+\tilde{\mu}^{(\kappa)}}{\left(\left\|\mathbf{x}^{(\kappa)}\right\|^{2}+\left\|\mathbf{y}^{(\kappa)}\right\|^{2}+\tilde{\mu}^{(\kappa)}\right)\left\|\mathbf{x}^{(\kappa)}\right\|^{2}}, \\
0<\quad & c\left(\mathbf{x}^{(\kappa)}, \mathbf{y}^{(\kappa)}, \mu^{(\kappa)}\right) \\
= & \frac{\left\|\mathbf{x}^{(\kappa)}\right\|^{2}}{\left(\left\|\mathbf{x}^{(\kappa)}\right\|^{2}+\left\|\mathbf{y}^{(\kappa)}\right\|^{2}+\tilde{\mu}^{(\kappa)}\right)\left(\left\|\mathbf{y}^{(\kappa)}\right\|^{2}+\tilde{\mu}^{(\kappa)}\right)}(20
\end{aligned}
$$

for $\tilde{\mu}^{(\kappa)}=\sigma+\sigma_{c} \mu^{(\kappa)}$. Let $\left(\mathbf{w}^{(\kappa)}, \mathcal{R}^{(\kappa)}, \boldsymbol{\alpha}^{(\kappa)}\right)$ be a feasible point for (13) that is found from the $(\kappa-1)$ th iteration, where $\boldsymbol{\alpha}^{(\kappa)} \triangleq\left(\alpha_{i, j}^{(\kappa)}\right)_{(i, j) \in \mathcal{S}}$ and $\mathcal{R}^{(\kappa)} \triangleq\left(\mathcal{R}_{i, j}^{(\kappa)}\right)_{(i, j) \in \mathcal{S}}$. Applying inequality (15) yields

$$
\begin{gathered}
\psi\left(\mathbf{h}_{i, i, j} \mathbf{w}_{i, p(j)}, \mathcal{L}_{i, p(j)}^{c}(\mathbf{w}), \alpha_{i, j}\right) \\
\geq \quad \Lambda\left(\mathbf{h}_{i, i, j} \mathbf{w}_{i, p(j)}, \mathcal{L}_{i, p(j)}^{c}(\mathbf{w}), 1 / \alpha_{i, j}\right)
\end{gathered}
$$

over the trust region

$$
\begin{aligned}
2 \Re\left\{\left(\mathbf{w}_{i, p(j)}^{(\kappa)}\right){ }^{H} \mathbf{h}_{i, i, p(j)}^{H} \mathbf{h}_{i, i, p(j)} \mathbf{w}_{i, p(j)}\right\} & \\
-\left\|\mathbf{h}_{i, i, p(j)} \mathbf{w}_{i, p(j)}^{(\kappa)}\right\|^{2} & >0,
\end{aligned}
$$

where the concave function $\Lambda\left(\mathbf{h}_{i, i, j} \mathbf{w}_{i, p(j)}, \mathcal{L}_{i, p(j)}^{c}(\mathbf{w}), 1 / \alpha_{i, j}\right)$ is defined from (17), (18)-(20) for $\mathbf{x}^{(\kappa)}=\mathbf{h}_{i, i, j} \mathbf{w}_{i, p(j)}^{(\kappa)}, \mathbf{y}^{(\kappa)}=\mathcal{L}_{i, p(j)}^{c}\left(\mathbf{w}^{(\kappa)}\right)$, and $\mu^{(\kappa)}=1 / \alpha_{i, j}^{(\kappa)}$. As such, the nonconvex constraint (9) is innerly approximated by the convex constraints (21) and

$$
\mathcal{R}_{i, p(j)} \leq \Lambda\left(\mathbf{h}_{i, i, j} \mathbf{w}_{i, p(j)}, \mathcal{L}_{i, p(j)}^{c}(\mathbf{w}), 1 / \alpha_{i, j}\right) .
$$

Analogously, the nonconvex constraints (10) and (11) are innerly approximated by the convex constraints

$$
\begin{array}{r}
\mathcal{R}_{i, p(j)} \leq \Lambda\left(\mathbf{h}_{i, i, p(j)} \mathbf{w}_{i, p(j)}, \mathcal{L}_{i, p(j)}^{e}(\mathbf{w}), 0\right), \\
\mathcal{R}_{i, j} \leq \Lambda\left(\mathbf{h}_{i, i, j} \mathbf{w}_{i, j}, \mathcal{L}_{i, j}^{c}(\mathbf{w}), \alpha_{i, j}\right), \\
2 \Re\left\{\left(\mathbf{w}_{i, p(j)}^{(\kappa)}\right)^{H} \mathbf{h}_{i, i, j}^{H} \mathbf{h}_{i, i, j} \mathbf{w}_{i, p(j)}\right\} \\
-\left\|\mathbf{h}_{i, i, j} \mathbf{w}_{i, p(j)}^{(\kappa)}\right\|^{2}>0, \\
2 \Re\left\{\left(\mathbf{w}_{i, j}^{(\kappa)}\right)^{H} \mathbf{h}_{i, i, j}^{H} \mathbf{h}_{i, i, j} \mathbf{w}_{i, j}\right\}-\left\|\mathbf{h}_{i, i, j} \mathbf{w}_{i, j}^{(\kappa)}\right\|^{2}>0,
\end{array}
$$

where the concave functions $\Lambda\left(\mathbf{h}_{i, i, p(j)} \mathbf{w}_{i, p(j)}, \mathcal{L}_{i, p(j)}^{e}(\mathbf{w}), 0\right)$ is defined from (17)-(20) for

$$
\left(\mathbf{x}^{(\kappa)}, \mathbf{y}^{(\kappa)}, \tilde{\mu}^{(\kappa)}\right)=\left(\mathbf{h}_{i, i, p(j)} \mathbf{w}_{i, p(j)}^{(\kappa)}, \mathcal{L}_{i, p(j)}^{e}\left(\mathbf{w}^{(\kappa)}\right), \sigma\right),
$$

while the concave functions $\Lambda\left(\mathbf{h}_{i, i, j} \mathbf{w}_{i, j}, \mathcal{L}_{i, j}^{c}(\mathbf{w}), 1 / \alpha_{i, j}\right)$ is defined from (17)-(20) for

$$
\left(\mathbf{x}^{(\kappa)}, \mathbf{y}^{(\kappa)}, \tilde{\mu}^{(\kappa)}\right)=\left(\mathbf{h}_{i, i, j} \mathbf{w}_{i, j}^{(\kappa)}, \mathcal{L}_{i, j}^{c}\left(\mathbf{w}^{(\kappa)}\right), \sigma+1 / \alpha_{i, j}^{(\kappa)}\right) .
$$

Next, by using the following approximation [7]

$$
\left|\mathbf{h}_{s, i, j}^{H} \mathbf{x}\right|^{2} \geq-\left|\mathbf{h}_{s, i, j}^{H} \mathbf{x}\right|^{2}+2 \Re\left\{\left(\mathbf{x}^{(\kappa)}\right)^{H} \mathbf{h}_{s, i, j} \mathbf{h}_{s, i, j}^{H} \mathbf{x}\right\},
$$

an inner convex approximation for the nonconvex EH constraint (13b) is given by

$$
\begin{array}{r}
\zeta_{i, j} \sum_{(s, \ell) \in \mathcal{S}}\left(2 \Re\left\{\left(\mathbf{w}_{s, \ell}^{(\kappa)}\right)^{H} \mathbf{h}_{s, i, j}^{H} \mathbf{h}_{s, i, j} \mathbf{w}_{s, \ell}\right\}\right. \\
\left.-\left|\mathbf{h}_{s, i, j} \mathbf{w}_{s, \ell}^{(\kappa)}\right|^{2}\right) \geq \frac{e_{i, j}^{\min }}{1-\alpha_{i, j}} .
\end{array}
$$

At the $\kappa$-th iteration, we solve the following convex quadratic optimization problem of computational complexity $\mathcal{O}\left(\left(2 K N\left(N_{t}+2\right)\right)^{3}(8 K N+N)\right)$ [37, p. 4] to generate the next feasible point $\left(\mathbf{w}^{(\kappa+1)}, \mathcal{R}^{(\kappa+1)}, \boldsymbol{\alpha}^{(\kappa+1)}\right)$ :

$$
\max _{\mathbf{w}, \boldsymbol{\alpha}, \mathcal{R}} \min _{(i, j) \in \mathcal{I} \times \mathcal{J}} \mathcal{R}_{i, j} \quad \text { s.t. } \quad(13 c),(13 d),(21)-(27) .
$$

Algorithm 1 outlines the steps to solve the throughput max-min optimization problem (13). Note that $\min _{(i, j) \in \mathcal{I} \times \mathcal{J}} \mathcal{R}_{i, j}^{(\alpha+1)}>\min _{(i, j) \in \mathcal{I} \times \mathcal{J}} \mathcal{R}_{i, j}^{(\alpha)}$ because $\left(\mathbf{w}^{(\kappa+1)}, \mathcal{R}^{(\kappa+1)}, \boldsymbol{\alpha}^{(\kappa+1)}\right)$ is the optimal solution of (28) while $\left(\mathbf{w}^{(\kappa)}, \mathcal{R}^{(\kappa)}, \boldsymbol{\alpha}^{(\kappa)}\right)$ is its feasible point. Therefore, Algorithm 1 is a path-following algorithm, which generates a sequence $\left\{\left(\mathbf{w}^{(\kappa)}, \mathcal{R}^{(\kappa)}, \boldsymbol{\alpha}^{(\kappa)}\right)\right\}$ of improved feasible points for (13). By using similar arguments to that shown in [5], [6], we can easily show that it converges at least to a locally optimal solution of (13), which satisfies the Karush-Kuhn-Tucker (KKT) optimality condition.

Since the EH constraint (13b) is nonconvex, locating an initial feasible point $\left(\mathbf{w}^{(0)}, \mathcal{R}^{(0)}, \boldsymbol{\alpha}^{(0)}\right)$ for (13) is a nonconvex problem, which is resolved via the iterations

$$
\begin{array}{r}
\max _{\mathbf{w}, \mathcal{R}, \boldsymbol{\alpha}} \min _{(i, j) \in \mathcal{I} \times \mathcal{J}_{c}}\left\{\frac{\mathcal{R}_{i, j}}{r_{0}}-1, \frac{\mathcal{R}_{i, p(j)}}{r_{0}}-1,\right. \\
\zeta_{i, j} \sum_{(s, \ell) \in \mathcal{S}}\left(2 \Re\left\{\left(\mathbf{w}_{s, \ell}^{(\kappa)}\right)^{H} \mathbf{h}_{s, i, j}^{H} \mathbf{h}_{s, i, j} \mathbf{w}_{s, l}\right\}\right. \\
\left.\left.-\left|\mathbf{h}_{s, i, j} \mathbf{w}_{s, l}^{(\kappa)}\right|^{2}\right)-\frac{e_{i, j}^{\min }}{1-\alpha_{i, j}}\right\} \\
\text { s.t. } \quad(13 \mathrm{c}),(13 \mathrm{~d}),(21)-(26)
\end{array}
$$

for a fixed $r_{0}>0^{2}$ till reaching a value more than or equal to 0 and thus the feasibility for (13).

Algorithm 1 PS-based algorithm for throughput max-min optimization problem (13)

1: Initialization: Set $\kappa:=0$ and initialize a feasible point $\left(\mathbf{w}^{(0)}, \mathcal{R}^{(0)}, \boldsymbol{\alpha}^{(0)}\right)$ for (13)

2: Repeat until convergence of the objective in (13): Solve the convex optimization problem (28) to generate the feasible point $\left(\mathbf{w}^{(\kappa+1)}, \mathcal{R}^{(\kappa+1)}, \boldsymbol{\alpha}^{(\kappa+1)}\right)$ for (13); Reset $\kappa:=\kappa+1$.

Next, we address the EE maximization problem (14), which is equivalent to the following minimization problem

$$
\min _{\mathbf{w}, \boldsymbol{\alpha}, \mathcal{R}} \frac{\xi \pi(\mathbf{w})+P_{c}}{\sum_{(i, j) \in \mathcal{I} \times \mathcal{J}} \mathcal{R}_{i, j}}
$$

s.t. $(9),(10),(11),(13 b),(13 c),(13 d),(14 b),(14 c)$. (30)

\footnotetext{
${ }^{2} r_{0}$ plays the role of good initial value of the max-min throughput
} 
The advantage of this transformation is that the objective function is already convex so there is no need of using Dinkelbach's iteration or approximating it. Actually, the Dinkelbach iteration for (14) still involves a nonconvex optimization problem, which is as computationally difficult as (14) itself (see e.g., [34]). At the $\kappa$-th iteration, we solve the following convex optimization problem of computational complexity $\mathcal{O}\left(\left(2 K N\left(N_{t}+2\right)\right)^{3}(10 K N+N)\right)$ to generate its next feasible point $\left(\mathbf{w}^{(\kappa+1)}, \mathcal{R}^{(\kappa+1)}, \boldsymbol{\alpha}^{(\kappa+1)}\right)$ :

$$
\begin{aligned}
& \min _{\mathbf{w}, \boldsymbol{\alpha}, \mathcal{R}} \frac{\xi \pi(\mathbf{w})+P_{c}}{\sum_{(i, j) \in \mathcal{I} \times \mathcal{J}} \mathcal{R}_{i, j}} \\
& \text { s.t. } \quad(13 c),(13 d),(14 b),(14 c),(21)-(27) .
\end{aligned}
$$

Algorithm 2 outlines a path-following computational procedure to solve the the energy-efficiency (EE) maximization problem (14). Like Algorithm 1, at least it converges to a locally optimal solution of (14), which satisfies the KKT optimality condition.

Locating a feasible point $\left(\mathbf{w}^{(0)}, \mathcal{R}^{(0)}, \boldsymbol{\alpha}^{(0)}\right)$ for the nonconvex constraints (13b), (9), (10), and (11) to initialize Algorithm 2 is resolved via the iterations

$$
\begin{array}{r}
\max _{\mathbf{w}, \mathcal{R}, \boldsymbol{\alpha}} \min _{(i, j) \in \mathcal{I} \times \mathcal{J}_{c}}\left\{\frac{\mathcal{R}_{i, j}}{r_{i, j}}-1, \frac{\mathcal{R}_{i, p(j)}}{r_{i, p(j)}}-1,\right. \\
\zeta_{i, j} \sum_{(s, \ell) \in \mathcal{S}}\left(2 \Re\left\{\left(\mathbf{w}_{s, \ell}^{(\kappa)}\right)^{H} \mathbf{h}_{s, i, j}^{H} \mathbf{h}_{s, i, j} \mathbf{w}_{s, l}\right\}\right. \\
\left.\left.-\left|\mathbf{h}_{s, i, j} \mathbf{w}_{s, l}^{(\kappa)}\right|^{2}\right)-\frac{e_{i, j}^{\min }}{1-\alpha_{i, j}}\right\} \\
\text { s.t. }(13 \mathrm{c}),(13 \mathrm{~d}),(21)-(26)
\end{array}
$$

till reaching a value more than or equal to 0 .

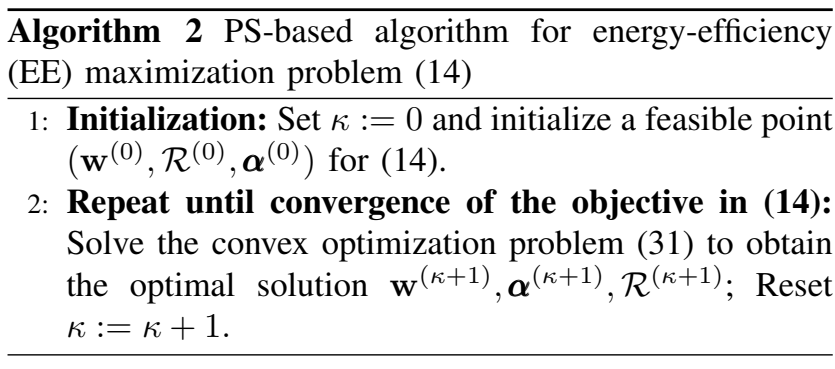

\section{TRANSMIT TS-BASED NOMA}

In a time-switching (TS) based system, a fraction of time $0<\rho<1$ is used for power transfer while the remaining fraction of time $1-\rho$ for information transfer, where $\rho$ is termed as TS ratio. For power transfer, we have to design the energy beamforming vectors $\mathbf{w}_{i, j}^{E}, \forall, i \in \mathcal{I}, j \in \mathcal{J}_{c}$. For $p_{i, j}\left(\mathbf{w}^{E}\right) \triangleq \sum_{(s, \ell) \in \mathcal{S}_{c}}\left|\mathbf{h}_{s, i, j} \mathbf{w}_{s, \ell}^{E}\right|^{2}$ and $\zeta_{i, j} \in(0,1)$ as the energy conversion efficiency for the $\mathrm{EH}$ receiver, the harvested energy by the cell-center user $\operatorname{UE}(i, j)$ is expressed as

$$
E_{i, j}\left(\mathbf{w}^{E}, \rho\right) \triangleq \rho \zeta_{i, j}\left(p_{i, j}\left(\mathbf{w}^{E}\right)\right) .
$$

Here, we assume a common TS ratio $\rho$ for all BSs, $i \in$ $\mathcal{I}$, where near-by users harvest energy through wireless signals not only from the serving BSs but also from the neighboring BSs. Let us denote $\mathbf{w}^{E} \triangleq\left[\mathbf{w}_{i, j}^{E}\right]_{i \in \mathcal{I}, j \in \mathcal{J}_{c}}$.

The remaining time $(1-\rho)$ will be used for information decoding by all users $\operatorname{UE}(i, j), i \in \mathcal{I}, j \in \mathcal{J}$. Let $\mathbf{w}^{I} \triangleq$ $\left[\mathbf{w}_{i, j}^{I}\right]_{i \in \mathcal{I}, j \in \mathcal{J}}$ define the information beamforming vectors and suppose that $\mathcal{R}_{i, p(j)}$ is the achievable rate by decoding $s_{i, p(j)}$. Recalling the definitions (6), (7), (12) and (8), we have

$$
\begin{gathered}
\mathcal{R}_{i, p(j)} \leq(1-\rho) \psi\left(\mathbf{h}_{i, i, j} \mathbf{w}_{i, p(j)}^{I}, \mathcal{L}_{i, p(j)}^{c}\left(\mathbf{w}^{I}\right), 0\right), \\
\mathcal{R}_{i, p(j)} \leq(1-\rho) \psi\left(\mathbf{h}_{i, i, p(j)} \mathbf{w}_{i, p(j)}^{I}, \mathcal{L}_{i, p(j)}^{e}\left(\mathbf{w}^{I}\right), 0\right) .
\end{gathered}
$$

After decoding $s_{i, p(j)}$, UE $(i, j), j \in \mathcal{J}_{c}$ decodes $s_{i, j}$. The achievable rate $\mathcal{R}_{i, j}$ by decoding $s_{i, j}$ is

$$
\mathcal{R}_{i, j} \leq(1-\rho) \psi\left(\mathbf{h}_{i, i, j} \mathbf{w}_{i, j}^{I}, \mathcal{L}_{i, j}^{c}\left(\mathbf{w}^{I}\right), 0\right) .
$$

In the following subsections, we present problem formulation and solution for throughput max-min optimization and energy-efficiency optimization.

\section{A. Throughput max-min Optimization}

Thus, the throughput max-min optimization problem for TS-based NOMA SWIPT system is given by

$$
\begin{array}{r}
\max _{\mathbf{w}^{I}, \mathbf{w}^{E}, \mathcal{R}, \rho} \min _{(i, j) \in \mathcal{I} \times \mathcal{J}} \mathcal{R}_{i, j} \quad \text { s.t. } \quad(34),(35),(36), \\
\zeta_{i, j}\left(p_{i, j}\left(\mathbf{w}^{E}\right)\right) \geq \frac{e_{i, j}^{\min }}{\rho}, i \in \mathcal{I}, j \in \mathcal{J}_{c}, \\
0<\rho<1, \\
\frac{1}{1-\rho} \sum_{j \in \mathcal{J}_{c}}\left\|\mathbf{w}_{i, j}^{E}\right\|^{2}+\sum_{j \in \mathcal{J}}\left\|\mathbf{w}_{i, j}^{I}\right\|^{2} \leq \\
\frac{P_{i}^{\max }}{1-\rho}+\sum_{j \in \mathcal{J}_{c}}\left\|\mathbf{w}_{i, j}^{E}\right\|^{2}, i \in \mathcal{I} \\
\left\|\mathbf{w}_{i, j}^{E}\right\|^{2} \leq P_{i}^{\max } i \in \mathcal{I}, j \in \mathcal{J}_{c} \\
\left\|\mathbf{w}_{i, j}^{I}\right\|^{2} \leq P_{i}^{\max } i \in \mathcal{I}, j \in \mathcal{J} .
\end{array}
$$

Note that $(37 d)$ is the equivalent reexpression of the sum power constraint

$$
\rho \sum_{j \in \mathcal{J}_{c}}\left\|\mathbf{w}_{i, j}^{E}\right\|^{2}+(1-\rho) \sum_{j \in \mathcal{J}}\left\|\mathbf{w}_{i, j}^{I}\right\|^{2} \leq P_{i}^{\max }, i \in \mathcal{I},
$$

while (37e) and (37f) set the limit of transmit power during any fraction of time.

Let $\left(\mathbf{w}^{I,(\kappa)}, \mathbf{w}^{E,(\kappa)}, \mathcal{R}^{(\kappa)}, \rho^{(\kappa)}\right)$ be a feasible point for (37) that is found from the $(\kappa-1)$ th iteration. By using the inequality $\left.x \leq 0.5\left(x^{2} / \bar{x}+\bar{x}\right) \forall x\right), \bar{x}>0$ [38, (78)] and (15), we can innerly approximate (34), (35) and (36) by

$$
\begin{gathered}
0.5 \frac{\left.\mathcal{R}_{i, p(j)}\right)^{2} / \mathcal{R}_{i, p(j)}^{(\kappa)}+\mathcal{R}_{i, p(j)}^{(\kappa)}}{1-\rho} \leq \\
\Lambda\left(\mathbf{h}_{i, i, j} \mathbf{w}_{i, p(j)}^{I}, \mathcal{L}_{i, p(j)}^{c}\left(\mathbf{w}^{I}\right), 0\right),
\end{gathered}
$$




$$
\begin{gathered}
0.5 \frac{\left(\mathcal{R}_{i, p(j)}\right)^{2} / \mathcal{R}_{i, p(j)}^{(\kappa)}+\mathcal{R}_{i, p(j)}^{(\kappa)}}{1-\rho} \leq \\
\Lambda\left(\mathbf{h}_{i, i, p(j)} \mathbf{w}_{i, p(j)}^{I}, \mathcal{L}_{i, p(j)}^{e}\left(\mathbf{w}^{I}\right), 0\right),
\end{gathered}
$$

and

$$
0.5 \frac{\left(\mathcal{R}_{i, j}\right)^{2} / \mathcal{R}_{i, j}^{(\kappa)}+\mathcal{R}_{i, j}^{(\kappa)}}{1-\rho} \leq \Lambda\left(\mathbf{h}_{i, i, j} \mathbf{w}_{i, j}^{I}, \mathcal{L}_{i, j}^{c}\left(\mathbf{w}^{I}\right), 0\right)
$$

over the trust regions

$$
\begin{aligned}
& 2 \Re\left\{\left(\mathbf{w}_{i, p(j)}^{I,(\kappa)}\right)^{H} \mathbf{h}_{i, i, p(j)}^{H} \mathbf{h}_{i, i, p(j)} \mathbf{w}_{i, p(j)}^{I}\right\} \\
&-\left\|\mathbf{h}_{i, i, p(j)} \mathbf{w}_{i, p(j)}^{I,(\kappa)}\right\|^{2}>0,(41 \mathrm{a}) \\
& 2 \Re\left\{\left(\mathbf{w}_{i, p(j)}^{I,(\kappa)}\right)^{H} \mathbf{h}_{i, i, j}^{H} \mathbf{h}_{i, i, j} \mathbf{w}_{i, p(j)}^{I}\right\} \\
&-\left\|\mathbf{h}_{i, i, j} \mathbf{w}_{i, p(j)}^{I,(\kappa)}\right\|^{2}>0,(41 \mathrm{~b}) \\
& 2 \Re\left\{\left(\mathbf{w}_{i, j}^{I,(\kappa)}\right)^{H} \mathbf{h}_{i, i, j}^{H} \mathbf{h}_{i, i, j} \mathbf{w}_{i, j}^{I}\right\} \\
&-\left\|\mathbf{h}_{i, i, j} \mathbf{w}_{i, j}^{I,(\kappa)}\right\|^{2}>0,(41 \mathrm{c})
\end{aligned}
$$

with the concave functions $\Lambda\left(\mathbf{h}_{i, i, j} \mathbf{w}_{i, p(j)}^{I}, \mathcal{L}_{i, p(j)}^{c}\left(\mathbf{w}^{I}\right), 0\right)$, $\Lambda\left(\mathbf{h}_{i, i, p(j)} \mathbf{w}_{i, p(j)}^{I}, \mathcal{L}_{i, p(j)}^{e}\left(\mathbf{w}^{I}\right), 0\right)$, and

$\Lambda\left(\mathbf{h}_{i, i, j} \mathbf{w}_{i, j}^{I}, \mathcal{L}_{i, j}^{c}\left(\mathbf{w}^{I}\right), 0\right)$ defined from (17), (18)-(20) for $\left(\mathbf{x}^{(\kappa)}, \mathbf{y}^{(\kappa)}, \tilde{\mu}^{(\kappa)}\right)=\left(\mathbf{h}_{i, i, j} \mathbf{w}_{i, p(j)}^{I,(\kappa)}, \mathcal{L}_{i, p(j)}^{c}\left(\mathbf{w}^{I,(\kappa)}\right), \sigma\right)$, $\left(\mathbf{x}^{(\kappa)}, \mathbf{y}^{(\kappa)}, \tilde{\mu}^{(\kappa)}\right)=\left(\mathbf{h}_{i, i, p(j)} \mathbf{w}_{i, p(j)}^{I,(\kappa)}, \mathcal{L}_{i, p(j)}^{e}\left(\mathbf{w}^{I,(\kappa)}\right), \sigma\right)$, and $\left(\mathbf{x}^{(\kappa)}, \mathbf{y}^{(\kappa)}, \tilde{\mu}^{(\kappa)}\right)=\left(\mathbf{h}_{i, i, j} \mathbf{w}_{i, j}^{I,(\kappa)}, \mathcal{L}_{i, j}^{c}\left(\mathbf{w}^{I,(\kappa)}\right), \sigma\right)$, respectively.

The EH constraint (37b) is innerly approximated by [7]

$$
\begin{aligned}
& \sum_{(s, \ell) \in \mathcal{S}_{c}}\left[2 \Re\left\{\left(\mathbf{w}_{s, \ell}^{E,(\kappa)}\right)^{H} \mathbf{h}_{s, i, j}^{H} \mathbf{h}_{s, i, j} \mathbf{w}_{s, \ell}^{E}\right\}\right. \\
& \left.-\left|\mathbf{h}_{i, i, j} \mathbf{w}_{s, \ell}^{E,(\kappa)}\right|^{2}\right] \geq \frac{e_{i, j}^{\min }}{\zeta_{i, j} \rho}, i \in \mathcal{I}, j \in \mathcal{J}_{c},
\end{aligned}
$$

while the power constraint (37d) is innerly approximated by [7]

$$
\begin{array}{r}
\frac{1}{1-\rho} \sum_{j \in \mathcal{J}_{c}}\left\|\mathbf{w}_{i, j}^{E}\right\|^{2}+\sum_{j \in \mathcal{J}}\left\|\mathbf{w}_{i, j}^{I}\right\|^{2} \leq \\
\left(\frac{2}{1-\rho^{(\kappa)}}-\frac{1-\rho}{\left(1-\rho^{(\kappa)}\right)^{2}}\right) P_{i}^{\max } \\
+\sum_{j \in \mathcal{J}}\left(2 \Re\left\{\left(\mathbf{w}_{i, j}^{E,(\kappa)}\right)^{H} \mathbf{w}_{i, j}^{E}\right\}-\left\|\mathbf{w}_{i, j}^{E,(\kappa)}\right\|^{2}\right) .
\end{array}
$$

In summary, at the $\kappa$ th iteration we solve the following convex optimization problem of computational complexity $\mathcal{O}\left(\left(2 K N\left(N_{t}+N_{t} / 2+1\right)+1\right)^{3}(9 K N+N+1)\right)$ to generate the feasible point $\left(\mathbf{w}^{I,(\kappa+1)}, \mathbf{w}^{E,(\kappa+1)}, \mathcal{R}^{(\kappa)}, \rho^{(\kappa+1)}\right)$ for (37):

$$
\begin{array}{ll} 
& \max _{\mathbf{w}^{E}, \mathbf{w}^{I}, \mathcal{R}, \rho} \min _{(i, j) \in \mathcal{I} \times \mathcal{J}} \mathcal{R}_{i, j} \\
\text { s.t. } & (37 c),(37 e),(37 f),(38)-(43) .
\end{array}
$$

Algorithm 3 outlines a path-following computational procedure to solve the throughput max-min optimization problem (37). Like Algorithm 1, it converges at least to a local optimal solution satisfying the KKT condition. To find an initial feasible point $\left(\mathbf{w}^{E,(0)}, \mathbf{w}^{I,(0)}, \rho^{(0)}, \mathcal{R}^{(0)}\right)$ of (37), we first fix $\rho^{(0)}$ and find $\left(\mathbf{w}^{E,(0)}, \mathbf{w}^{I,(0)}\right)$ by randomly generating $N_{t} \times 1$ complex vectors followed by their normalization to satisfy (37d), (37e), and (37f). We then find $\left(\mathbf{w}^{E,(0)}, \mathbf{w}^{I,(0)}, \mathcal{R}^{(0)}\right)$ via the iterations

$$
\begin{gathered}
\max _{\mathbf{w}^{E}, \mathbf{w}^{I}, \gamma>0} \gamma \text { s.t. } \quad(37 e),(37 f), \\
\zeta_{i, j} \sum_{(s, \ell) \in \mathcal{S}_{c}}\left(2 \Re\left\{\left(\mathbf{w}_{s, \ell}^{E,(\kappa)}\right)^{H} \mathbf{h}_{s, i, j}^{H} \mathbf{h}_{s, i, j} \mathbf{w}_{s, l}^{E}\right\}\right. \\
\left.\quad-\left|\mathbf{h}_{s, i, j} \mathbf{w}_{s, l}^{E,(\kappa)}\right|^{2}\right) \geq \gamma \frac{e_{i, j}^{\min }}{\rho^{(0)}},(i, j) \in \mathcal{I} \times \mathcal{J}_{c} \\
\rho^{(0)} \sum_{j \in \mathcal{J}_{c}}\left\|\mathbf{w}_{i, j}^{E}\right\|^{2}+\left(1-\rho^{(0)}\right) \sum_{j \in \mathcal{J}}\left\|\mathbf{w}_{i, j}^{I}\right\|^{2} \\
\leq P_{i}^{\max }, \quad i \in \mathcal{I}, \\
\frac{r_{0}}{1-\rho^{(0)}} \gamma \leq \Lambda\left(\mathbf{h}_{i, i, j} \mathbf{w}_{i, p(j)}^{I}, \mathcal{L}_{i, p(j)}^{c}\left(\mathbf{w}^{I}\right), 0\right), \\
\frac{r_{0}}{1-\rho^{(0)}} \gamma \leq \Lambda\left(\mathbf{h}_{i, i, p(j)} \mathbf{w}_{i, p(j)}^{I}, \mathcal{L}_{i, p(j)}^{e}\left(\mathbf{w}^{I}\right), 0\right), \\
\frac{r_{0}}{1-\rho^{(0)}} \gamma \leq \Lambda\left(\mathbf{h}_{i, i, j} \mathbf{w}_{i, j}^{I}, \mathcal{L}_{i, j}^{c}\left(\mathbf{w}^{I}\right), 0\right),
\end{gathered}
$$

till reaching a value more than or equal to 1 , for fixed $\rho^{(0)}$ and $r_{0}{ }^{3}$

$\overline{\text { Algorithm } 3 \text { Transmit TS-based algorithm for throughput }}$ max-min optimization problem (37)

1: Initialization: Set $\kappa:=0$ and initialize a feasible point $\left(\mathbf{w}^{E,(0)}, \mathbf{w}^{I,(0)}, \mathcal{R}^{(0)}, \rho^{(0)}\right)$ for (37).

2: Repeat until convergence of the objective in (37): Solve the convex optimization problem (44) to obtain the optimal solution $\left(\mathbf{w}^{E,(\kappa+1)}, \mathbf{w}^{I,(\kappa+1)}, \mathcal{R}^{(\kappa+1)}, \rho^{(\kappa+1)}\right) ; \quad$ Reset $\kappa:=\kappa+1$.

\section{B. Energy-Efficiency Optimization}

Next, we address the following energy-efficiency maximization problem

$$
\begin{aligned}
& \max _{\mathbf{w}^{E}, \mathbf{w}^{I}, \mathcal{R}, \rho} \frac{\sum_{(i, j) \in \mathcal{I} \times \mathcal{J}} \mathcal{R}_{i, j}}{\xi\left[\rho \pi_{E}\left(\mathbf{w}^{E}\right)+(1-\rho) \pi_{I}\left(\mathbf{w}^{I}\right)\right]+P_{c}} \\
& \text { s.t. }(14 b),(14 c),(34)-(36),(37 b)-(37 f) \text {, }
\end{aligned}
$$

where $\pi_{E}\left(\mathbf{w}^{E}\right)=\sum_{i \in \mathcal{I}} \sum_{j \in \mathcal{J}_{c}}\left\|\mathbf{w}_{i, j}^{E}\right\|^{2}$ and $\pi_{I}\left(\mathbf{w}^{I}\right)=$ $\sum_{i \in \mathcal{I}} \sum_{j \in \mathcal{J}}\left\|\mathbf{w}_{i, j}^{I}\right\|^{2}$. Note that we define $\rho \pi_{E}\left(\mathbf{w}^{E}\right)+$ $(1-\rho) \pi_{I}\left(\mathbf{w}^{I}\right)$ to differentiate from $\pi(\mathbf{w})$ in (30). For this network, it is more appropriate to use $(1-\rho) \pi_{I}\left(\mathbf{w}^{I}\right)$ for information delivery.

In contrast to the objective function in (14), the power consumption function in the denominator of the objective

${ }^{3}$ Like that in (29), $r_{0}$ plays the role of good initial value of the max-min throughput. The initial point $\left(\mathbf{w}^{E,(0)}, \mathbf{w}^{I,(0)}\right)$ for $(45)$ is any feasible point for the convex constraints (37e) and (37f), Simulation results in Sec. IV show that $\rho^{(0)}=0.2$ is a good choice. 
function in (46) is no longer convex, making equivalently transformed problem like (14) no longer useful. Nevertheless, we now also develop another iterative procedure, where (46) is seen no more computationally difficult than the throughput optimization problem (37).

Let $\left(\mathbf{w}^{E,(\kappa)}, \mathbf{w}^{I,(\kappa)}, \mathcal{R}^{(\kappa)}, \rho^{(\kappa)}\right)$ be a feasible point for (46) that is found from the $(\kappa-1)$ th iteration and

$$
\begin{aligned}
& t^{(\kappa)} \triangleq \\
& \frac{\sum_{(i, j) \in \mathcal{I} \times \mathcal{J}} \mathcal{R}_{i, j}^{(\kappa)}}{\xi\left[\rho^{(\kappa)} \pi_{E}\left(\mathbf{w}^{E,(\kappa)}\right)+\left(1-\rho^{(\kappa)}\right) \pi_{I}\left(\mathbf{w}^{I,(\kappa)}\right)\right]+P_{c}}= \\
& \frac{\sum_{(i, j) \in \mathcal{I} \times \mathcal{J}} \mathcal{R}_{i, j}^{(\kappa)} /\left(1-\rho^{(\kappa)}\right)}{\xi\left[\frac{\pi_{E}\left(\mathbf{w}^{E,(\kappa)}\right)}{\left(1-\rho^{(\kappa)}\right)-1}+\xi \pi_{I}\left(\mathbf{w}^{I,(\kappa)}\right)\right]+\frac{P_{c}}{1-\rho^{(\kappa)}}}
\end{aligned}
$$

At the $\kappa$ th iteration we address the problem

$$
\begin{array}{r}
\max _{\mathbf{w}^{E}, \mathbf{w}^{I}, \mathcal{R}, \rho} \frac{\sum_{(i, j) \in \mathcal{I} \times \mathcal{J}} \mathcal{R}_{i, j}}{1-\rho}-t^{(\kappa)}\left[\xi\left(\frac{1}{1-\rho}-1\right)\right. \\
\left.\times \pi_{E}\left(\mathbf{w}^{E}\right)+\xi \pi_{I}\left(\mathbf{w}^{I}\right)+\frac{P_{c}}{1-\rho}\right] \\
\text { s.t. } \quad(14 b),(14 c),(34)-(36),(37 b)-(37 f) .
\end{array}
$$

Substituting $x=\sum_{(i, j) \in \mathcal{I} \times \mathcal{J}} \mathcal{R}_{i, j}, \bar{x}=\sum_{(i, j) \in \mathcal{I} \times \mathcal{J}} \mathcal{R}_{i, j}^{(\kappa)}$, and $t=1-\rho, \bar{t}=1-\rho^{(\kappa)}$ into the following inequality

$$
\frac{x}{t} \geq 2 \frac{\sqrt{\bar{x}}}{\bar{t}} \sqrt{x}-\frac{\bar{x}}{\bar{t}^{2}} t \quad \forall x>0, \bar{x}>0, t>0, \bar{t}>0,
$$

the proof of which is given by Appendix B, the first term in the objective of (47) is lower bounded by the concave function

$$
\begin{aligned}
f^{(\kappa)}(\mathcal{R}, \rho) \triangleq & 2 \frac{\sqrt{\sum_{(i, j) \in \mathcal{I} \times \mathcal{J}} \mathcal{R}_{i, j}^{(\kappa)}}}{1-\rho^{(\kappa)}} \sqrt{\sum_{(i, j) \in \mathcal{I} \times \mathcal{J}} \mathcal{R}_{i, j}} \\
& -\frac{\sum_{(i, j) \in \mathcal{I} \times \mathcal{J}} \mathcal{R}_{i, j}^{(\kappa)}}{\left(1-\rho^{(\kappa)}\right)^{2}}(1-\rho) .
\end{aligned}
$$

The second term $g(\mathbf{w}, \rho) \triangleq \xi\left(\frac{1}{1-\rho}-1\right) \pi_{E}\left(\mathbf{w}^{E}\right)+$ $\xi \pi_{I}\left(\mathbf{w}^{I}\right)+\frac{P_{c}}{1-\rho}$ in the objective of (47) is upper bounded by the convex function

$$
\begin{aligned}
& g^{(\kappa)}(\mathbf{w}, \rho) \triangleq \frac{1}{(1-\rho)} \xi \pi_{E}\left(\mathbf{w}^{E}\right)+\xi \pi_{I}\left(\mathbf{w}^{I}\right)+\frac{P_{c}}{1-\rho} \\
& -\xi \sum_{i \in \mathcal{I}} \sum_{j \in \mathcal{J}_{c}}\left(2 \Re\left\{\left(\mathbf{w}_{i, j}^{E,(\kappa)}\right)^{H} \mathbf{w}_{i, j}^{E}\right\}-\left\|\mathbf{w}_{i, j}^{E,(\kappa)}\right\|^{2}\right),
\end{aligned}
$$

because $\|\mathbf{x}\|^{2} \geq 2 \Re\left\{\left(\mathbf{x}^{(\kappa)}\right)^{H} \mathbf{x}\right\}-\left\|\mathbf{x}^{(\kappa)}\right\|^{2}$.

Thus, we solve the following convex optimization problem of computational complexity $\mathcal{O}\left(\left(2 K N\left(N_{t}+N_{t} / 2+1\right)+1\right)^{3}(11 K N+N+1)\right) \quad$ to generate $\left(\mathbf{w}^{E,(\kappa+1)}, \mathbf{w}^{I,(\kappa+1)}, \mathcal{R}^{(\kappa+1)}, \rho^{(\kappa+1)}\right)$ :

$$
\begin{array}{r}
\max _{\mathbf{w}^{E}, \mathbf{w}^{I}, \mathcal{R}, \rho}\left[f^{(\kappa)}(\mathcal{R}, \rho)-t^{(\kappa)} g^{(\kappa)}(\mathbf{w}, \rho)\right] \\
\text { s.t. } \quad(14 b),(14 c),(38)-(43) .
\end{array}
$$

Algorithm 4 outlines the steps to solve the energyefficiency (EE) maximization problem (46). Locating an initial feasible point $\left(\mathbf{w}^{E,(0)}, \mathbf{w}^{I,(0)}, \rho^{(0)}, \mathcal{R}^{(0)}\right)$ for (46)

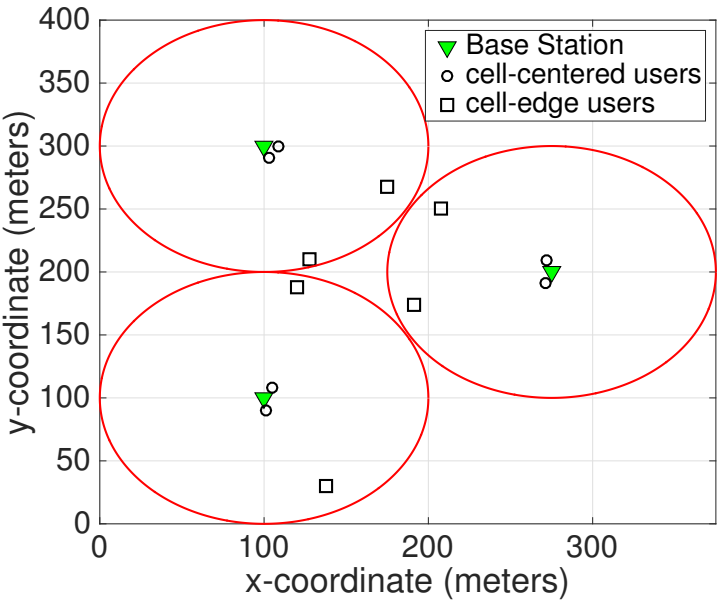

Fig. 2. A multicell network setup used in our numerical examples.

is resolved via iterations (45) with $r_{i, p(j)}\left(r_{i, j}\right.$, resp.) replacing $r_{0}$ in $(45 \mathrm{~d})$ and $(45 \mathrm{e})((45 \mathrm{f})$, resp.) till reaching a value greater than or equal to 1 .

Algorithm 4 Transmit TS-based algorithm for energyefficiency (EE) maximization problem (46)

1: Initialization: Set $\kappa:=0$ and initialize a feasible point $\left(\mathbf{w}^{E,(0)}, \mathbf{w}^{I,(0)}, \rho^{(0)}, \mathcal{R}^{(0)}\right)$ for (46).

2: Repeat until convergence of the objective in (46): Solve the convex optimization problem (49) to obtain the optimal solution $\left(\mathbf{w}^{E,(\kappa+1)}, \mathbf{w}^{I,(\kappa+1)}, \mathcal{R}^{(\kappa+1)}, \rho^{(\kappa+1)}\right) ; \quad$ Reset $\kappa:=\kappa+1$.

\section{Simulation Results}

To analyze the proposed algorithms through simulations, a network topology as shown in Fig. 2 is set up. There are $N=3$ cells and $2 K=4$ UEs per cell with two placed close to the BS and the remaining two placed near cell-edges. These 12 users are served over the same time and frequency. Other users are allocated different frequency band or different time for communication. The cell radius is set to be 100 meters, where, near-by users are placed about the distance of 10 meters from the serving BS while cell-edge users are placed about the distance of $80-90$ meters from the serving BS.

The channel $\mathbf{h}_{s, i, j}$ from BS $s \in \mathcal{I}$ to $\operatorname{UE}(i, j)$ at a distance of $d$ meters is generated as $\mathbf{h}_{s, i, j}=$ $\sqrt{10^{-\sigma_{\mathrm{PL}} / 10}} \tilde{\mathbf{h}}_{s, i, j}$, where $\sigma_{\mathrm{PL}}=30+10 \beta \log _{10}(d)$ is the path-loss in $\mathrm{dB}$ (the path-loss model is consistent with dense user deployment settings and wireless energy harvesting requirements [25]), $\beta$ is the path-loss exponent, and $\tilde{\mathbf{h}}_{s, i, j}$ is the normalized Rayleigh fading channel gain (for $s=i$ while $j \in \mathcal{J}_{e}$ or $s \neq i$, i.e., channel between BS and its own cell-edge users or channel between BS and users in the neigboring cells) or $\tilde{\mathbf{h}}_{s, i, j}$ is the Rician fading channel 
gain with Rician factor of $10 \mathrm{~dB}$ (for $s=i$ while $j \in \mathcal{J}_{c}$, i.e., channel between BS and its own cell-centered users). We set the path-loss exponents $\beta=\beta_{1}=3$ for the former case and $\beta=\beta_{2}=2$ for the later case. Different values for path-loss exponents have been proposed for different type of users in the literature too [39], [40]. Considering the adopted path-loss model and practical simulation setup, the power of the received signal at the cell-center UEs passes the threshold minimum power requirement $(-21 \mathrm{dBm}$ with $13 \mathrm{~nm}$ CMOS technology [1]) to carry out meaningful EH. For simplicity, set $e_{i, j}^{\min } \equiv e^{\min }$ for the energy harvesting thresholds, $\zeta_{i, j} \equiv \zeta, \quad \forall i, j$ for the energy harvesting conversion, $P_{i}^{\max } \equiv P^{\max }, \forall i$. Further, $e^{\min }=-20 \mathrm{dBm}$ for the energy harvesting threshold, $\zeta=0.5$ for the energy conversion efficiency, and $P^{\max }=35 \mathrm{dBm}$ are set (unless stated otherwise). The bandwidth is set to $B=20 \mathrm{MHz}$, the carrier frequency is set to $2 \mathrm{GHz}$, and unless stated otehrwise, the power spectral density of additive white Gaussian noise, $\frac{\sigma}{B}$ or $\frac{\sigma_{c}}{B}$, is set to $-174 \mathrm{dBm} / \mathrm{Hz}$. For energy efficiency maximization problems (14) and (46), the threshold rate $r_{i, j}=0.5 \mathrm{bits} / \mathrm{sec} / \mathrm{Hz} \forall i, j$ (unless stated otherwise), the power amplifier efficiency $1 / \xi=0.2$, power dissipation at each transmit antenna $P_{A}=0.6 \mathrm{~W}$ $(27.78 \mathrm{dBm})$, and circuit power consumption $P_{\text {cir }}=2.5 \mathrm{~W}$ $(33.97 \mathrm{dBm})$ are set [41], [42]. The convex solver CVX [43] is used.

\section{A. Results for throughput max-min optimization problems (13) and (37):}

On average (running 100 simulations and averaging over random channel realizations), the PS-based Algorithm 1 requires 30.05 iterations, while TS-based Algorithm 3 requires 18.25 iterations for convergence.

Fig. 3 plots the optimized max-min rate for varying number of BS-antennas and fixed BS power budget $P^{\max }=35$ $\mathrm{dBm}$ by solving the power splitting (PS)-based problem (13) and the time switching (TS)-based problem (37). As expected, the rate increases by increasing the number of antennas at the BS. Fig. 3 shows that the TS-based Algorithm 3 outperforms the PS-based Algorithm 1 in terms of achievable rate and the corresponding performance gap increases by increasing the number of antennas mounted on the BS. In the TS-based model, the presence of more antennas helps both information and energy beamforming vectors to scale their performance more progressively as compared to the PS-based model with optimizing only the information beamforming vectors. Fig. 4 plots the optimized worst user rate for varying values of BS transmit power budget $P^{\max }$ and fixed number of BS-antennas $N_{t}=6$, while solving the same power splitting (PS)-based problem (13) and time switching (TS)-based problem (37). Fig. 4 shows that increasing the transmit power budget raises the level of achievable rate, however, the increase diminishes at higher values of transmit power budget, e.g., there is a marginal improvement in the rate when the transmit power budget is increased from $P^{\max }=43 \mathrm{dBm}$ to $47 \mathrm{dBm}$. Similarly to Fig. 3, Fig. 4 shows that the TSbased Algorithm 3 outperforms the PS-based Algorithm 1 in terms of achievable rate.

Fig. 5 plots the optimized worst user rate for varying values of noise power spectral density $\frac{\sigma}{B}$ (in $\mathrm{dBm} / \mathrm{Hz}$ ) and fixed value of transmit power budget $P^{\max }=35 \mathrm{dBm}$ and BS antennas $N_{t}=6$, while solving the same power splitting (PS)-based problem (13) and time switching (TS)based problem (37). Fig. 5 shows that, as expected, increasing the noise variance decreases the level of achievable rate. However, there is a minor decrease in the achievable rate for PS-based receiver. Therefore, though the TS-based Algorithm 3 outperforms the PS-based Algorithm 1 in terms of achievable rate, the corresponding performance gap decreases by increasing the noise variance. Under a similar simulation setup, Fig. 6 plots the optimized worst user rate for varying values of path-loss exponent $\beta_{1}$, showing that increasing the path-loss exponent increases the level of achievable rate. Indeed, it is an interesting result as it goes against the usual expectation of decrease in the achievable rate with the increase in path-loss exponent. The unexpected trend of increase in the achievable rate with the increase in the path-loss exponent is due to the consideration of multicell setup. The intercell interference decreases with the increase in the path-loss exponent, which boosts the achievable rate of the affected cell-edge users.

Remark 1: Figs. 3-6 show that the TS-based Algorithm 3 outperforms the PS-based Algorithm 1 in terms of spectral efficiency. This is because for the TS-based Algorithm 3 , we separately optimize the beamforming vectors for information transmission and energy harvesting, which results in better design compared to the case of PS-based Algorithm 1, where same beamforming vector is used for both information transmission and energy harvesting.

\section{B. Results for energy efficiency maximization problems} (14) and (46):

On average, the PS-based Algorithm 2 requires 15.05 iterations, while the TS-based Algorithm 4 requires 15.7 iterations before convergence.

Fig. 7 plots the optimized energy efficiency (EE) for a varying number of BS-antennas and fixed BS power budget $P^{\max }=35 \mathrm{dBm}$, while solving power splitting (PS)-based problem (14) and time switching (TS)-based problem (46). As expected, the EE increases by increasing the number of antennas at the BS. We can also observe from Fig. 7 that the TS-based Algorithm 4 outperforms the PS-based Algorithm 2 in terms of achievable EE and the performance gap is more than 1 bit/Joule. Next, Fig. 8 plots the energy efficiency for varying values of BS transmit power budget $P^{\max }$ and fixed number of BS-antennas $N_{t}=4$, while solving the same power splitting (PS)-based problem (14) and time switching (TS)-based problem (46). 


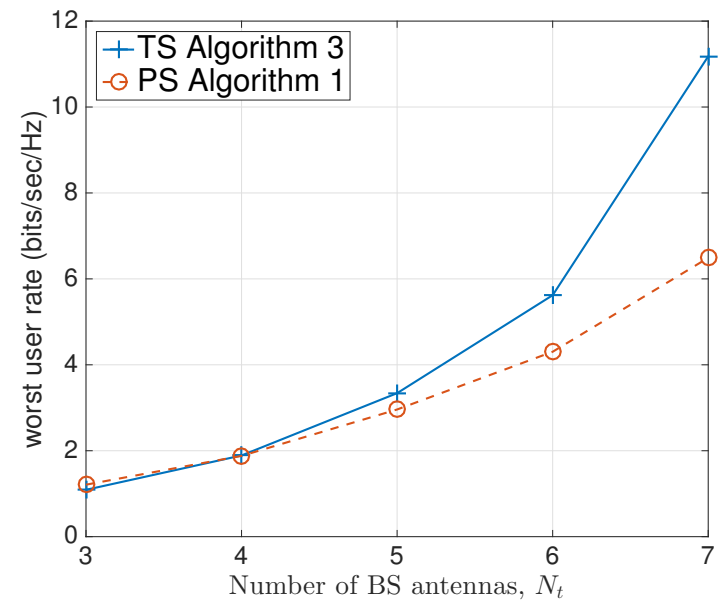

Fig. 3. Optimized worst user rate for varying number of antennas and fixed BS power budget $P^{\max }=35 \mathrm{dBm}$, by solving the power splitting (PS)-based problem (13) and the time switching (TS)-based problem (37).

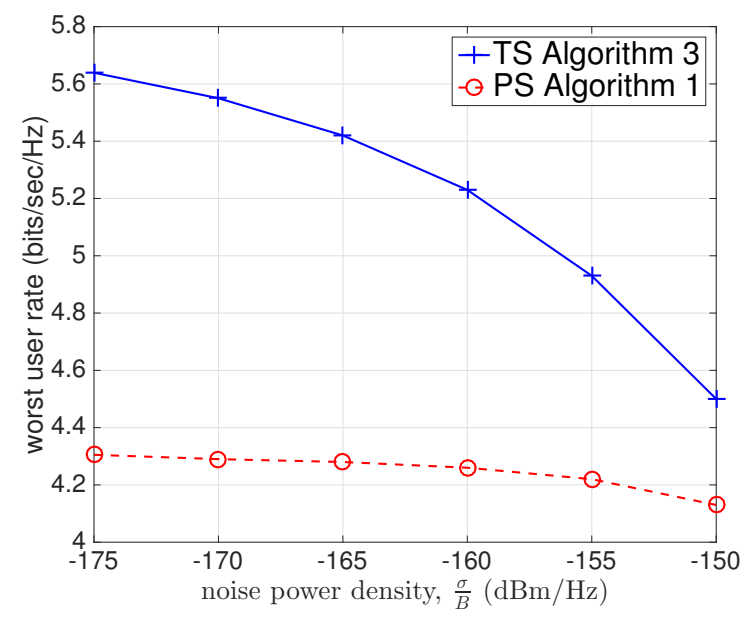

Fig. 5. Optimized worst user rate for varying values of noise power spectral density $\frac{\sigma}{B}$ and fixed value of $P^{\max }=35 \mathrm{dBm}$ and BS antennas $N_{t}=6$ by solving the power splitting (PS)-based problem (13) and the time switching (TS)-based problem (37).

Fig. 8 shows that for TS-based implementation, increasing the transmit power budget raises the level of achievable $\mathrm{EE}$, however, for PS-based implementation, there is almost no improvement in EE when the transmit power budget is increased from $P^{\max }=35 \mathrm{dBm}$ to $45 \mathrm{dBm}$.

Fig. 9 plots the optimized energy efficiency for different values of noise power spectral density $\frac{\sigma}{B}$ (in $\mathrm{dBm} / \mathrm{Hz}$ ) and fixed value of transmit power budget $P^{\max }=35 \mathrm{dBm}$ and BS antennas $N_{t}=4$, while solving the power splitting (PS)-based problem (14) and time switching (TS)-based problem (46). Fig. 9 shows that, as expected, EE decreases by increasing the noise variance. In addition, the TS-based Algorithm 4 outperforms the PS-based Algorithm 2 in terms of EE. Nevertheless, this performance gap decreases

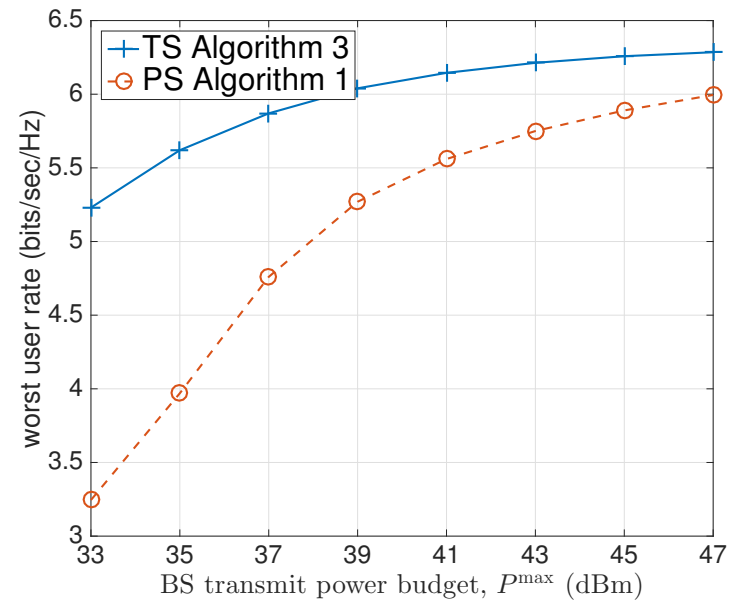

Fig. 4. Optimized worst user rate for varying values of BS transmit power budget $P^{\max }$ and fixed value of $N_{t}=6$, by solving the power splitting (PS)-based problem (13) and the time switching (TS)-based problem (37).

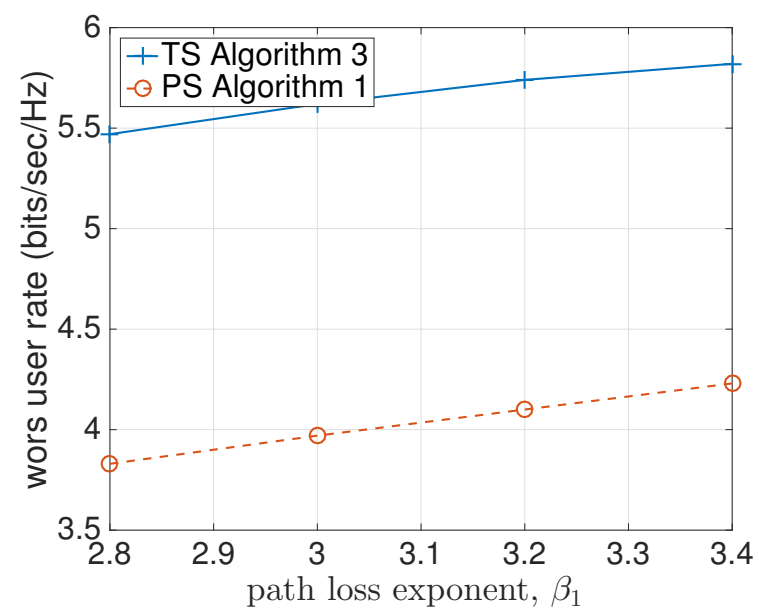

Fig. 6. Optimized worst user rate for varying values of path-loss exponent $\beta_{1}$ and fixed value of $P^{\max }=35 \mathrm{dBm}$ and $\mathrm{BS}$ antennas $N_{t}=6$ by solving the power splitting (PS)-based problem (13) and the time switching (TS)-based problem (37).

with the increase of noise variance.

\section{Comparison with orthogonal multiple access (OMA):}

As mention in Section I, the problem of EE maximization with multi-antenna beamforming in an EH-enabled NOMA network has not been addressed in the literature before. In addition, the existing research did not consider "transmit-TS" approach for throughput max-min optimization of the EH-enabled NOMA network. Therefore, in this subsection, we compare the performance of our proposed algorithms with that obtained by OMA implementation.

Fig. 10 plots the optimized worst user rate for varying values of BS transmit power budget $P^{\max }$ and fixed number of BS-antennas $N_{t}=6$ by solving the same power splitting 


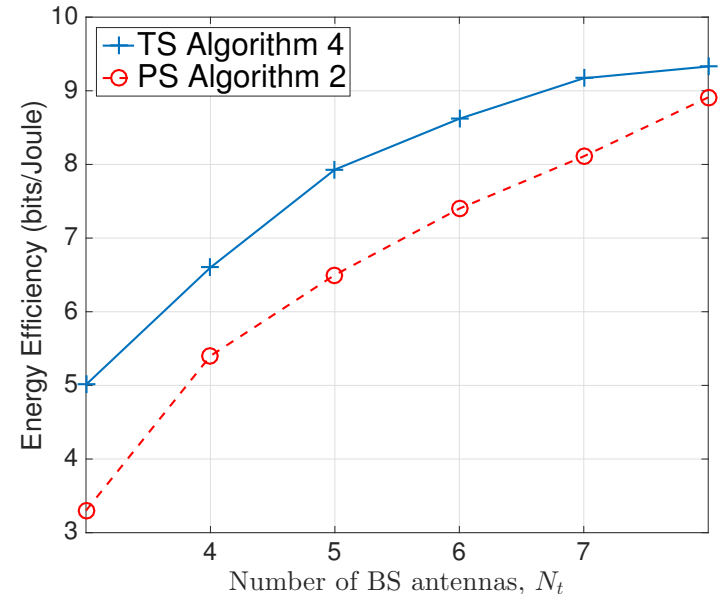

Fig. 7. Optimized energy efficiency for varying number of antennas and fixed BS power budget $P^{\max }=35 \mathrm{dBm}$, while solving power splitting (PS)-based problem (14) and time switching (TS)-based problem (46).

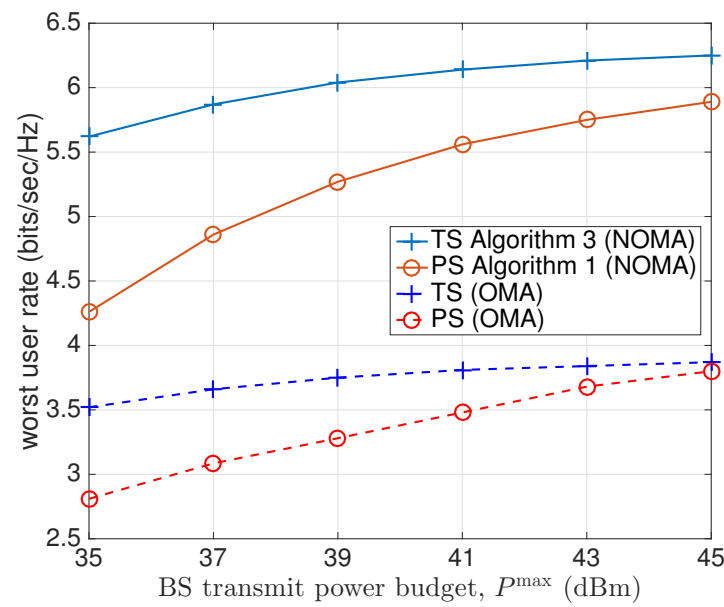

Fig. 10. Optimized worst user rate for varying values of BS transmit power budget $P^{\max }$ and fixed value of $N_{t}=6$, while solving power splitting (PS)-based NOMA problem (13), time switching (TS)-based NOMA problem (37), and their OMA counterparts.

(PS)-based NOMA problem (13) and the time switching (TS)-based NOMA problem (37), and their orthogonal multiple access (OMA) counterparts. In parallel, Fig. 11 plots the EE for varying values of BS transmit power budget $P^{\max }$ and fixed number of BS-antennas $N_{t}=4$ and threshold rate $r_{i, j}=0.1 \mathrm{bits} / \mathrm{sec} / \mathrm{Hz}$ (different from previous EE plots) by solving the power splitting (PS)based NOMA problem (14), the time switching (TS)based NOMA problem (46), and their OMA counterparts. We have to choose a smaller threshold rate $r_{i, j}=0.1$ bits/sec/Hz because implementation with OMA scheme fails to simultaneously satisfy both the higher threshold rate and the EH constraint for all simulations. We can clearly

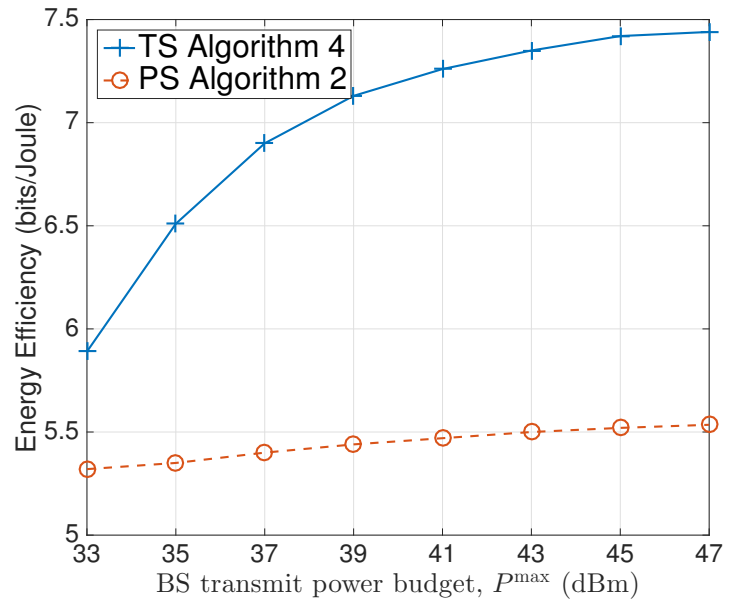

Fig. 8. Optimized energy efficiency for varying values of noise power spectral density $\frac{\sigma}{B}$ and fixed BS transmit power budget $P^{\max }=35$ and fixed value of $N_{t}=4$, while solving power splitting (PS)-based problem (14) and time switching (TS)-based problem (46)

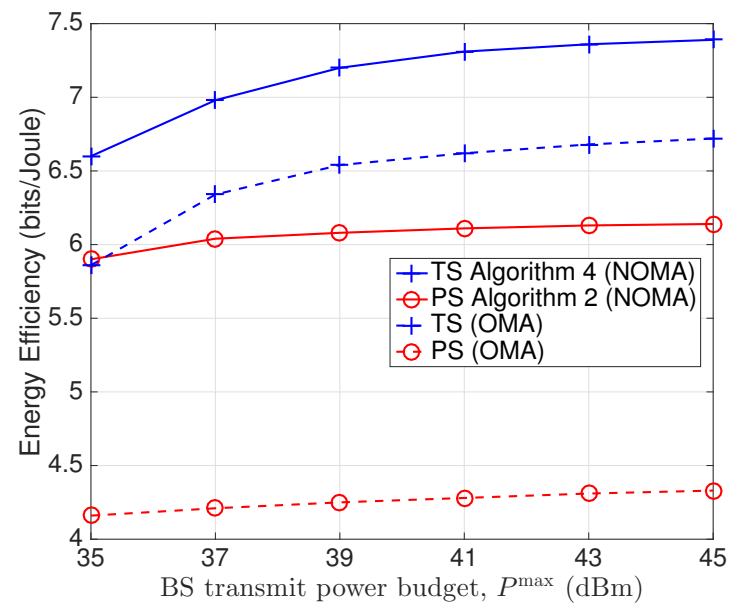

Fig. 11. Optimized energy efficiency for varying values of BS transmit power budget $P^{\max }$ and fixed value of $N_{t}=4$ and threshold rate $r_{i, j}=0.1 \mathrm{bits} / \mathrm{sec} / \mathrm{Hz}$ (different from previous EE plots), while solving power splitting (PS)-based NOMA problem (14), time switching (TS)based NOMA problem (46), and their OMA counterparts.

observe from Figs. 10 and 11 that NOMA implementation outperforms OMA implementation, in terms of both, throughput and energy efficiency, respectively.

\section{CONCLUSIONS}

This paper has considered an energy harvesting based NOMA system, where transmit-TS approach is employed to realize both wireless energy harvesting and information decoding at the nearly-located users. We have formulated two important problems of worst-user throughput maximization and energy efficiency maximization under power constraint and energy harvesting constraints at the nearly-located users. For these problems, the optimization 


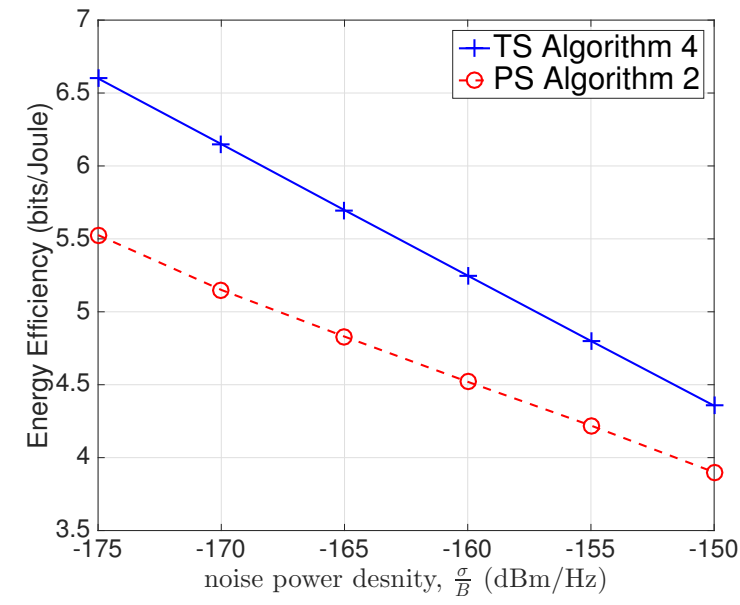

Fig. 9. Optimized energy efficiency for varying values of of noise variances $\sigma$ and fixed value of $P^{\max }=35 \mathrm{dBm}$ and BS antennas $N_{t}=4$, while solving power splitting (PS)-based problem (14) and time switching (TS)-based problem (46)

objective and energy harvesting constraints are highly nonconvex. To address this, we have developed efficient pathfollowing algorithms to solve the two problems. We have also proposed algorithms for the case if conventional PSbased approach is used for energy harvesting. Our numerical results confirmed that the proposed transmit-TS approach clearly outperforms PS approach in terms of both, throughput and energy efficiency. One possible future research direction is to consider many user-pairs per cell and jointly optimize beamforming vectors and user-pairing strategy.

\section{APPENDIX A: PROOF FOR (15)}

Define the function $f(x, y) \triangleq \ln \left(x^{-1}+y^{-1}\right)$ which is convex in $x>0$ and $y>0$ [44]. Then $f(x, y) \geq$ $f\left(x^{(\kappa)}, y^{(\kappa)}\right)+\left\langle\nabla f\left(x^{(\kappa)}, y^{(\kappa)}\right),(x, y)-\left(x^{(\kappa)}, y^{(\kappa)}\right)\right\rangle$ for all $x>0, y>0, x^{(\kappa)}>0, y^{(\kappa)}>0$ [45], which means that

$$
\begin{aligned}
\ln \left(\frac{1}{x}+\frac{1}{y}\right) \geq & \ln \left(\frac{1}{x^{(\kappa)}}+\frac{1}{y^{(\kappa)}}\right)+1 \\
& -\frac{1}{x^{(\kappa)}+y^{(\kappa)}}\left(\frac{y^{(\kappa)}}{x^{(\kappa)}} x+\frac{x^{(\kappa)}}{y^{(\kappa)}} y\right) .
\end{aligned}
$$

Substituting $x=\|\mathbf{x}\|^{2}, y=\|\mathbf{y}\|^{2}+\sigma+\sigma_{c} \mu, x^{(\kappa)}=$ $\left\|\mathbf{x}^{(\kappa)}\right\|^{2}$, and $y^{(\kappa)}=\left\|\mathbf{y}^{(\kappa)}\right\|^{2}+\sigma+\sigma \mu^{(\kappa)}$, we obtain

$$
\begin{array}{r}
\ln \left(\left(\|\mathbf{x}\|^{2}\right)^{-1}+\left(\|\mathbf{y}\|^{2}+\sigma+\sigma_{c} \mu\right)^{-1}\right) \\
\ln \left(\left(\left\|\mathbf{x}^{(\kappa)}\right\|^{2}\right)^{-1}+\left(\left\|\mathbf{y}^{(\kappa)}\right\|^{2}+\tilde{\mu}^{(\kappa)}\right)^{-1}\right)+1 \\
-\frac{1}{\left\|\mathbf{x}^{(\kappa)}\right\|^{2}+\left\|\mathbf{y}^{(\kappa)}\right\|^{2}+\tilde{\mu}^{(\kappa)}}\left(\frac{\left\|\mathbf{y}^{(\kappa)}\right\|^{2}+\tilde{\mu}^{(\kappa)}}{\left\|\mathbf{x}^{(\kappa)}\right\|^{2}}\|\mathbf{x}\|^{2}\right. \\
\left.+\frac{\left\|\mathbf{x}^{(\kappa)}\right\|^{2}}{\left\|\mathbf{y}^{(\kappa)}\right\|^{2}+\tilde{\mu}^{(\kappa)}}\left(\|\mathbf{y}\|^{2}+\tilde{\mu}^{(\kappa)}\right)\right),
\end{array}
$$

where $\tilde{\mu}^{(\kappa)} \triangleq \sigma+\sigma_{c} \mu^{(\kappa)}$. Next, as the functions $\ln (1 / x)$ and $\|\mathbf{x}\|^{2}$ are convex, it is true that

$$
\begin{aligned}
\ln \left(\frac{1}{x}\right) & \geq \ln \left(\frac{1}{x^{(\kappa)}}\right)-\frac{x-x^{(\kappa)}}{x^{(\kappa)}}, \\
\|\mathbf{x}\|^{2} & \geq 2 \Re\left\{\left(\mathbf{x}^{(\kappa)}\right)^{H} \mathbf{x}\right\}-\left\|\mathbf{x}^{(\kappa)}\right\|^{2} .
\end{aligned}
$$

By substituting $\|\mathbf{x}\|^{2}$ and $\left\|\mathbf{x}^{(\kappa)}\right\|^{2}$ in place of $\frac{1}{x}$ and $\frac{1}{x^{(\kappa)}}$ in (52), we have the following inequality:

$$
\ln \left(\|\mathbf{x}\|^{2}\right) \geq \ln \left(\left\|\mathbf{x}^{(\kappa)}\right\|^{2}\right)+1-\frac{\left\|\mathbf{x}^{(\kappa)}\right\|^{2}}{2 \Re\left\{\left(\mathbf{x}^{(\kappa)}\right)^{H} \mathbf{x}\right\}-\left\|\mathbf{x}^{(\kappa)}\right\|^{2}}
$$

over the trust region (16). Combining (51) and (54) leads to (15)-(17).

\section{APPENDIX B: PROOF FOR (48)}

As function $g(x, t) \triangleq x^{2} / t$ is convex in $x>0$ and $t>0$, it is true that $[45] \frac{x^{2}}{t} \geq g(\bar{x}, \bar{t})+\langle\nabla g(\bar{x}, \bar{t}),(x, t)-(\bar{x}, \bar{t})\rangle$ $=2 \frac{\bar{x}}{t} x-\frac{\bar{x}^{2}}{t^{2}} t$. Inequality (48) then follows by resetting $x \rightarrow \sqrt{x}$ and $\bar{x} \rightarrow \sqrt{\bar{x}}$.

\section{REFERENCES}

[1] X. Lu, P. Wang, D. Niyato, D. I. Kim, and Z. Han, "Wireless networks with RF energy harvesting: A contemporary survey," IEEE Commun. Surveys Tuts., vol. 17, pp. 757-789, 2015.

[2] A. A. Nasir, H. D. Tuan, D. T. Ngo, S. Durrani, and D. I. Kim, "Path-following algorithms for beamforming and signal splitting in RF energy harvesting networks," IEEE Commun. Letters, vol. 20, no. 8, pp. 1687-1690, Aug 2016.

[3] V. D. Nguyen, T. Q. Duong, H. D. Tuan, O. S. Shin, and H. V. Poor, "Spectral and energy efficiencies in full-duplex wireless information and power transfer," IEEE Trans. Commun., vol. 65, no. 5, pp. 22202233, May 2017.

[4] H. H. M. Tam, H. D. Tuan, A. A. Nasir, T. Q. Duong, and H. V. Poor, "MIMO energy harvesting in full-duplex multi-user networks," IEEE Trans. Wirel. Commun., vol. 16, no. 5, pp. 3282-3297, May 2017.

[5] A. A. Nasir, H. D. Tuan, T. Q. Duong, and H. V. Poor, "Secrecy rate beamforming for multicell networks with information and energy harvesting," IEEE Trans. Signal Process., vol. 65, no. 3, pp. 677689, 2017.

[6] A. A. Nasir, H. D. Tuan, D. T. Ngo, T. Q. Duong, and H. V. Poor, "Beamforming design for wireless information and power transfer systems: Receive power-splitting vs transmit time-switching," IEEE Trans. Commun., vol. 65, no. 2, pp. 876-889, 2017.

[7] A. A. Nasir, H. D. Tuan, T. Q. Duong, and H. V. Poor, "Secure and energy-efficient beamforming for simultaneous information and energy transfer," IEEE Trans. Wirel. Commun., vol. 16, no. 11, pp. 7523-7537, Nov 2017.

[8] Y. Saito, Y. Kishiyama, A. Benjebbour, T. Nakamura, A. Li, and K. Higuchi, "Non-orthogonal multiple access (NOMA) for cellular future radio access," in Proc. IEEE Veh. Technol. Conf. (VTC Spring), June 2013, pp. 1-5.

[9] W. Shin, M. Vaezi, B. Lee, D. J. Love, J. Lee, and H. V. Poor, "Nonorthogonal multiple access in multi-cell networks: Theory, performance, and practical challenges," IEEE Commun. Mag., vol. 55, no. 10, pp. 176-183, Oct. 2017.

[10] L. Dai, B. Wang, Z. Ding, Z. Wang, S. Chen, and L. Hanzo, "A survey of non-orthogonal multiple access for 5G," IEEE Commun. Surveys Tuts., vol. 20, no. 3, pp. 2294-2323, thirdquarter 2018.

[11] V. D. Nguyen, H. D. Tuan, T. Q. Duong, H. V. Poor, and O. S. Shin, "Precoder design for signal superposition in MIMO-NOMA multicell networks," IEEE J. Select. Areas Commun., vol. 35, no. 12, pp. 2681-2695, Dec 2017. 
[12] P. D. Diamantoulakis, K. N. Pappi, Z. Ding, and G. K. Karagiannidis, "Wireless-powered communications with non-orthogonal multiple access," IEEE Trans. Wirel. Commun., vol. 15, no. 12, pp. 8422-8436, Dec 2016.

[13] H. Chingoska, Z. Hadzi-Velkov, I. Nikoloska, and N. Zlatanov, "Resource allocation in wireless powered communication networks with non-orthogonal multiple access," IEEE Wirel. Commun. Lett., vol. 5, no. 6, pp. 684-687, Dec 2016.

[14] M. Song and M. Zheng, "Energy efficiency optimization for wireless powered sensor networks with non-orthogonal multiple access," IEEE Sensors Letters, vol. PP, no. 99, pp. 1-1, 2018.

[15] M. Moltafet, P. Azmi, N. Mokari, M. R. Javan, and A. Mokdad, "Optimal and fair energy efficient resource allocation for energy harvesting enabled-PD-NOMA based hetnets," IEEE Trans. Wirel. Commun., vol. PP, no. 99, pp. 1-1, 2018.

[16] Y. Wang, Y. Wu, F. Zhou, Z. Chu, Y. Wu, and F. Yuan, "Multiobjective resource allocation in a NOMA cognitive radio network with a practical non-linear energy harvesting model," IEEE Access, vol. PP, no. 99, pp. 1-1, 2017.

[17] Z. Yang, Y. Pan, W. Xu, R. Guan, Y. Wang, and M. Chen, "Energy efficient resource allocation for machine-to-machine communications with NOMA and energy harvesting," in Proc IEEE INFOCOM, May 2017, pp. 145-150.

[18] Z. Yang, W. Xu, Y. Pan, C. Pan, and M. Chen, "Energy efficient resource allocation in machine-to-machine communications with multiple access and energy harvesting for IoT," IEEE Internet of Things J., vol. PP, no. 99, pp. 1-1, 2017.

[19] M. Ashraf, A. Shahid, J. W. Jang, and K. G. Lee, "Energy harvesting non-orthogonal multiple access system with multi-antenna relay and base station," IEEE Access, vol. 5, pp. 17660-17 670, 2017.

[20] W. Han, J. Ge, and J. Men, "Performance analysis for NOMA energy harvesting relaying networks with transmit antenna selection and maximal-ratio combining over Nakagami-m fading," IET Coтmun., vol. 10, no. 18, pp. 2687-2693, 2016.

[21] Y. Zhang, J. Ge, and E. Serpedin, "Performance analysis of a 5G energy-constrained downlink relaying network with non-orthogonal multiple access," IEEE Trans. Wirel. Commun., vol. 16, no. 12, pp. 8333-8346, Dec 2017.

[22] Z. Yang, Z. Ding, P. Fan, and N. Al-Dhahir, "The impact of power allocation on cooperative non-orthogonal multiple access networks with swipt," IEEE Trans. Wirel. Commun., vol. 16, no. 7, pp. 4332 4343, July 2017.

[23] Y. Liu, Z. Ding, M. Elkashlan, and H. V. Poor, "Cooperative nonorthogonal multiple access with simultaneous wireless information and power transfer," IEEE J. Select. Areas Commun., vol. 34, no. 4, pp. 938-953, April 2016.

[24] Y. Xu, C. Shen, Z. Ding, X. Sun, S. Yan, G. Zhu, and Z. Zhong, "Joint beamforming and power-splitting control in downlink cooperative SWIPT NOMA systems," IEEE Trans. Signal Process., vol. 65, no. 18 , pp. $4874-4886$, Sept. 2017.

[25] T. N. Do, D. B. da Costa, T. Q. Duong, and B. An, "Improving the performance of cell-edge users in MISO-NOMA systems using TAS and SWIPT-based cooperative transmissions," IEEE Trans. Green Commun. Netw., vol. PP, no. 99, pp. 1-1, 2017.

[26] W. Guo and Y. Wang, "Cooperative non-orthogonal multiple access with energy harvesting," Information, vol. 8, no. 3, 2017.

[27] J. Gong and X. Chen, "Achievable rate region of non-orthogonal multiple access systems with wireless powered decoder," IEEE J. Select. Areas Commun., vol. 35, no. 12, pp. 2846-2859, Dec 2017.

[28] G. He, L. Li, X. Li, W. Chen, L. L. Yang, and Z. Han, "Secrecy sum rate maximization in NOMA systems with wireless information and power transfer," in Proc IEEE Int. Conf. Wirel. Commun. Signal Process. (WCSP), Oct 2017, pp. 1-6.

[29] P. Deng, B. Wang, W. Wu, and T. Guo, "Transmitter design in MISO-NOMA system with wireless-power supply," IEEE Commun. Lett., vol. 22, no. 4, pp. 844-847, Apr. 2018.

[30] F. Zhou, Z. Chu, H. Sun, R. Q. Hu, and L. Hanzo, "Artificial noise aided secure cognitive beamforming for cooperative MISO-NOMA using SWIPT," IEEE J. Select. Areas Commun., pp. 1-1, 2018.

[31] A. H. Phan, H. D. Tuan, H. H. Kha, and D. T. Ngo, "Nonsmooth optimization for efficient beamforming in cognitive radio multicast transmission," vol. 60, no. 6, pp. 2941-2951, Jun. 2012

[32] R. L. G. Cavalcante, S. Stanczak, M. Schubert, A. Eisenlatter, and U. Turke, "Toward energy-efficienct 5G wireless communications technologies," IEEE Signal Process. Mag., vol. 13, no. 11, pp. 2434, Nov. 2014.

[33] A. Zappone, L. Sanguinetti, G. Bacci, E. A. Jorswieck, and M. Debbah, "Energy-efficient power control: A look at 5G wirel. technologies," IEEE Trans. Signal Process., vol. 64, no. 7, pp. 1668-1683, Apr. 2016.

[34] S. Buzzi, C. L. I, T. E. Klein, H. V. Poor, C. Yang, and A. Zappone, "A survey of energy-efficient techniques for $5 \mathrm{G}$ networks and challenges ahead," IEEE J. Select. Areas Commun., vol. 34, no. 4, pp. 697-709, April 2016.

[35] Z. Ding, P. Fan, and H. V. Poor, "Impact of user pairing on 5G nonorthogonal multiple-access downlink transmissions," IEEE Trans. Veh. Technol., vol. 65, no. 8, pp. 6010-6023, Aug. 2016.

[36] E. Bjornson and E. Jorswieck, Optimal Resource Allocation in Coordinated Multi-cell Systems. Now Publishers, 2013.

[37] D. Peaucelle, D. Henrion, and Y. Labit, "Users guide for SeDuMi interface 1.03," 2002. [Online]. Available: http://homepages.laas.fr/ peaucell/software/sdmguide.pdf

[38] Z. Sheng, H. D. Tuan, A. A. Nasir, T. Q. Duong, and H. V. Poor, "Power allocation for energy efficiency and secrecy of interference wireless networks," IEEE Trans. Wirel. Commun., vol. 17, pp. 3737 3751, Jun. 2018.

[39] Y. Liu, Z. Qin, M. Elkashlan, A. Nallanathan, and J. A. McCann, "Non-orthogonal multiple access in large-scale heterogeneous networks," IEEE J. Select. Areas Commun., vol. 35, no. 12, pp. $2667-$ 2680, Dec 2017.

[40] A. Ghazanfari, H. Tabassum, and E. Hossain, "Ambient RF energy harvesting in ultra-dense small cell networks: performance and trade-offs," IEEE Wirel. Communications, vol. 23, no. 2, pp. 38 45, April 2016.

[41] M. Imran, E. Katranaras, G. Auer, O. Blume, V. Giannini, Y. J. I. Godor, M. Olsson, D. Sabella, P. Skillermark, and W. Wajda, "Analysis of the reference systems, areas of improvements and target breakdown," EARTH Project Deliverable D, vol. 2, Jun 2011.

[42] S. Leng, D. W. K. Ng, N. Zlatanov, and R. Schober, "Multi-objective beamforming for energy-efficient SWIPT systems," in Proc IEEE ICNC, Feb 2016, pp. 1-7.

[43] M. Grant and S. Boyd, "CVX: Matlab software for disciplined convex programming, version 2.1," http://cvxr.com/cvx, Mar. 2014.

[44] H. M. T. Ho, H. D. Tuan, D. T. Ngo, T. Q. Duong, and H. V. Poor, "Joint load balancing and interference management for smallcell heterogeneous networks with limited backhaul capacity," IEEE Trans. Wirel. Commun., vol. 16, no. 2, pp. 872-884, Feb. 2017.

[45] H. Tuy, Convex Analysis and Global Optimization (second edition). Springer International, 2016.

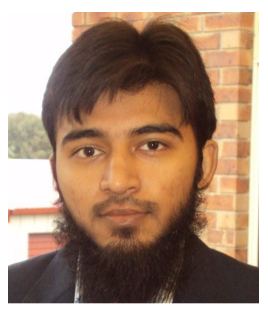

Ali Arshad Nasir (S'09-M'13) is an Assistant Professor in the Department of Electrical Engineering, King Fahd University of Petroleum and Minerals (KFUPM), Dhahran, KSA. Previously, he held the position of Assistant Professor in the School of Electrical Engineering and Computer Science (SEECS) at National University of Sciences \& Technology (NUST), Paksitan, from 2015-2016. He received his Ph.D. in telecommunications engineering from the Australian $\mathrm{Na}-$ tional University (ANU), Australia in 2013 and worked there as a Research Fellow from 2012-2015. His research interests are in the area of signal processing in wireless communication systems. $\mathrm{He}$ is an Associate Editor for IEEE Canadian Journal of Electrical and Computer Engineering. 


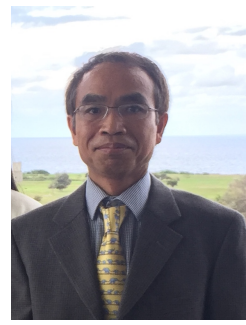

Hoang Duong Tuan received the Diploma (Hons.) and Ph.D. degrees in applied mathematics from Odessa State University, Ukraine, in 1987 and 1991, respectively. He spent nine academic years in Japan as an Assistant Professor in the Department of Electronic-Mechanical Engineering, Nagoya University, from 1994 to 1999, and then as an Associate Professor in the Department of Electrical and Computer Engineering, Toyota Technological Institute, Nagoya, from 1999 to 2003. He was a Professor with the School of Electrical Engineering and Telecommunications, University of New South Wales, from 2003 to 2011. He is currently a Professor with the School of Electrical and Data Engineering, University of Technology Sydney. He has been involved in research with the areas of optimization, control, signal processing, wireless communication, and biomedical engineering for more than 20 years.

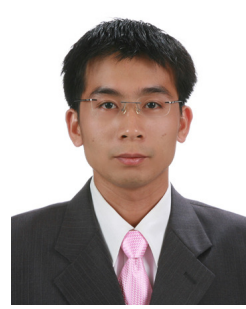

Trung Q. Duong (S'05, M'12, SM'13) received his Ph.D. degree in Telecommunications Systems from Blekinge Institute of Technology (BTH), Sweden in 2012. Currently, he is with Queen's University Belfast (UK), where he was a Lecturer (Assistant Professor) from 2013 to 2017 and a Reader (Associate Professor) from 2018. His current research interests include Internet of Things (IoT), wireless communications, molecular communications, and signal processing. He is the author or co-author of $330+$ technical papers published in scientific journals (196 articles) and presented at international conferences (125 papers).

Dr. Duong currently serves as an Editor for the IEEE TRANSACTIONS ON WIRELESS COMMUNICATIONS, IEEE TRANSACTIONS ON COMMUNICATIONS, IET COMMUNICATIONS, and a Lead Senior Editor for IEEE Communications LetTers. He was awarded the Best Paper Award at the IEEE Vehicular Technology Conference (VTC-Spring) in 2013, IEEE International Conference on Communications (ICC) 2014, IEEE Global Communications Conference (GLOBECOM) 2016, and IEEE Digital Signal Processing Conference (DSP) 2017. He is the recipient of prestigious Royal Academy of Engineering Research Fellowship (20162021) and has won a prestigious Newton Prize 2017.

Mérouane Debbah (S'01-M'04-SM'08-F'15) received the M.Sc. and Ph.D. degrees from the Ecole Normale Suprieure Paris-Saclay, France. In 1996, he joined the Ecole Normale Suprieure Paris-Saclay. He was with Motorola Labs, Saclay, France, from 1999 to 2002, and also with the Vienna Research Center for Telecommunications, Vienna, Austria, until 2003. From 2003 to 2007, he was an Assistant Professor with the Mobile Communications Department, Institut Eurecom, Sophia Antipolis, France. From 2007 to 2014, he was the Director of the Alcatel-Lucent Chair on Flexible Radio. Since 2007, he has been a Full Professor with CentraleSupelec, Gif-sur-Yvette, France. Since 2014, he has been a Vice-President of the Huawei France Research Center and the Director of the Mathematical and Algorithmic Sciences Lab. He has managed 8 EU projects and more than 24 national and international projects. His research interests lie in fundamental mathematics, algorithms, statistics, information, and communication sciences research. He was a recipient of the ERC Grant MORE (Advanced Mathematical Tools for Complex Network Engineering) from 2012 to 2017. He received 20 best paper awards, among which the 2015 IEEE Communications Society Leonard G. Abraham Prize, the 2016 IEEE Communications Society Best Tutorial Paper Award, and the 2018 IEEE Marconi Prize Paper Award. He is an Associate Editorin-Chief of the journal Random Matrix: Theory and Applications. He was an Associate Area Editor and Senior Area Editor of the IEEE Transactions on Signal Processing from 2011 to 2013 and from 2013 to 2014 , respectively. 